\title{
Some asymptotics for cranks
}

\author{
by \\ O-YeAT CHAN (Urbana, IL)
}

\section{INTRODUCTION}

In 1944 F. J. Dyson [8] defined the rank of a partition to be the largest part minus the number of parts. If we let $N(r, m, n)$ be the number of partitions of $n$ with rank congruent to $r$ modulo $m$, then it was conjectured by Dyson, and proved in 1954 by A. O. L. Atkin and H. P. F. Swinnerton-Dyer [5], that for all $k$,

$$
N(k, 5,5 n+4)=\frac{p(5 n+4)}{5}, \quad N(k, 7,7 n+5)=\frac{p(7 n+5)}{7} .
$$

These immediately imply two of the famous congruences of Ramanujan for the partition function, $p(5 n+4) \equiv 0(\bmod 5)$ and $p(7 n+5) \equiv 0(\bmod 7)$. However, the corresponding statement for the prime 11, namely, $11 N(k, 11$, $11 n+6)=p(11 n+6)$, is false. Dyson therefore conjectured the existence of the crank of a partition, a new statistic for which the corresponding formula would exist and hold for the prime 11. This crank was discovered by G. E. Andrews and F. G. Garvan in 1987, and is defined as follows.

DEFINITION 1.1. For a partition $\pi$ of a positive integer $n$, let $\lambda(\pi)$ be the largest part of $\pi$, let $\mu(\pi)$ be the number of ones in $\pi$, and let $\nu(\pi)$ be the number of parts of $\pi$ larger than $\mu(\pi)$. Then the $\operatorname{crank} c(\pi)$ is defined as

$$
c(\pi)= \begin{cases}\lambda(\pi) & \text { if } \mu(\pi)=0, \\ \nu(\pi)-\mu(\pi) & \text { if } \mu(\pi)>0 .\end{cases}
$$

If we define, for $n>1, M(m, n)$ to be the number of partitions of $n$ with crank $m$, and for $n \leq 1$, define $M(0,1)=-1, M(0,0)=M(1,1)=$ $M(-1,1)=1$, and $M(m, 0)=M(m, 1)=0$ otherwise, then it can be shown [2] that the generating function for $M(m, n)$ is given by

$$
\sum_{m=-\infty}^{\infty} \sum_{n=0}^{\infty} M(m, n) a^{m} q^{n}=\frac{(q ; q)_{\infty}}{(a q ; q)_{\infty}(q / a ; q)_{\infty}},
$$

2000 Mathematics Subject Classification: Primary 11P55; Secondary 11P82, 11P83. 
where, as usual,

$$
(a ; q)_{\infty}:=\prod_{n=0}^{\infty}\left(1-a q^{n}\right)
$$

and $|q|<1$.

Although Ramanujan probably had no knowledge of the cranks discovered by Andrews and Garvan, on page 179 of his Lost Notebook [11], he defines a function $F(q)$ and coefficients $\lambda_{n}$ by

$$
F(q):=F_{a}(q):=\frac{(q ; q)_{\infty}}{(a q ; q)_{\infty}(q / a ; q)_{\infty}}=: \sum_{n=0}^{\infty} \lambda_{n} q^{n} .
$$

Comparing (1.3) with (1.1) we immediately see that

$$
\lambda_{n}=\sum_{m=-\infty}^{\infty} M(m, n) a^{m} .
$$

On pages 179 and 180 of his Lost Notebook, Ramanujan lists ten tables of values of $n$ for which $\lambda_{n}$ satisfies certain congruence properties. In [7], Berndt et al. showed that these congruences are equivalent to having the coefficients of certain theta functions take on specific values. To understand these equivalent formulations, let us first define Ramanujan's general theta function.

Definition 1.2. For $|a b|<1$, let

$$
f(a, b):=\sum_{n=-\infty}^{\infty} a^{n(n-1) / 2} b^{n(n+1) / 2}
$$

and let the function $f(-q)$ be the case where $a=-q$ and $b=-q^{2}$. That is,

$$
f(-q):=f\left(-q,-q^{2}\right)
$$

The equivalent formulations of Ramanujan's tables are now summarized in Table 1.

One should note that Ramanujan missed the value $n=214$ in table 3 .

Berndt et al., in [7], also made the following conjecture regarding the functions

$$
\frac{f(-q) f\left(-q^{2}\right)}{f\left(-q^{4}\right)}, \frac{f\left(-q^{2}\right) f\left(-q^{3}\right)}{f\left(-q^{6}\right)}, \frac{f\left(-q^{6},-q^{10}\right)}{\left(-q^{4} ; q^{4}\right)_{\infty}}, q \frac{f\left(-q^{2},-q^{14}\right)}{\left(-q^{4} ; q^{4}\right)_{\infty}}, \frac{f^{2}(-q)}{f\left(-q^{3}\right)}
$$

ConjeCtURE 1.1. For each of the functions in (1.6), there exists a modulus a such that for each $0 \leq b<a$, the coefficients of $q^{a n+b}$ of that function are monotonic and strictly monotonic for sufficiently large $n$, according to Table 2. 
Table 1

\begin{tabular}{|c|c|c|c|}
\hline Table no. & Function & $N$ & $\begin{array}{l}\text { Values of } n \text { for which the coefficient } \\
\text { of } q^{n} \text { is equal to } N\end{array}$ \\
\hline 1 & $\frac{f\left(-q^{6},-q^{10}\right)}{\left(-q^{4} ; q^{4}\right)_{\infty}}+q \frac{f\left(-q^{2},-q^{14}\right)}{\left(-q^{4} ; q^{4}\right)_{\infty}}$ & 0 & $\begin{array}{l}2,8,9,10,11,15,19,21,22,25,26, \\
27,28,30,31,34,40,42,45,46,47,50, \\
55,57,58,59,62,66,70,74,75,78,79, \\
86,94,98,106,110,122,126,130,142, \\
154,158,170,174,206\end{array}$ \\
\hline 2 & $\frac{f\left(-q^{6},-q^{10}\right)}{\left(-q^{4} ; q^{4}\right)_{\infty}}$ & 1 & $\begin{array}{l}14,16,18,24,32,48,56,72,82,88,90 \\
104,114,138,146,162,178,186,194 \\
202,210,218,226,234,242,250,266\end{array}$ \\
\hline 3 & $\frac{f\left(-q^{6},-q^{10}\right)}{\left(-q^{4} ; q^{4}\right)_{\infty}}$ & -1 & $\begin{array}{l}4,6,12,20,36,38,44,52,54,60,68 \\
76,92,102,118,134,150,166,182,190 \\
214,222,238,254,270,286,302\end{array}$ \\
\hline 4 & $q \frac{f\left(-q^{2},-q^{14}\right)}{\left(-q^{4} ; q^{4}\right)_{\infty}}$ & 1 & $\begin{array}{l}1,7,17,23,33,39,41,49,63,71,73 \\
81,87,89,95,105,111,119,121,127 \\
143,159\end{array}$ \\
\hline 5 & $q \frac{f\left(-q^{2},-q^{14}\right)}{\left(-q^{4} ; q^{4}\right)_{\infty}}$ & -1 & $\begin{array}{l}3,5,13,29,35,37,43,51,53,61,67 \\
69,77,83,85,91,93,99,107,115,123, \\
139,155\end{array}$ \\
\hline 6 & $\frac{f(-q) f\left(-q^{2}\right)}{f\left(-q^{4}\right)}$ & 0 & $11,15,21$ \\
\hline 7 & $\frac{f\left(-q^{2}\right) f\left(-q^{3}\right)}{f\left(-q^{6}\right)}$ & 0 & $\begin{array}{l}1,6,8,13,14,17,19,22,23,25,33,34 \\
37,44,46,55,58,61,82\end{array}$ \\
\hline 8 & $\frac{f\left(-q^{2}\right) f\left(-q^{3}\right)}{f\left(-q^{6}\right)}$ & 1 & $\begin{array}{l}5,7,10,11,12,18,24,29,30,31,35 \\
41,42,43,47,49,53,54,59,67,71,73 \\
85,91,97,109\end{array}$ \\
\hline 9 & $\frac{f\left(-q^{2}\right) f\left(-q^{3}\right)}{f\left(-q^{6}\right)}$ & -1 & $\begin{array}{l}2,3,4,9,15,16,20,21,26,27,28,32 \\
38,39,40,52,56,62,64,68,70,76,94 \\
106,118,130\end{array}$ \\
\hline 10 & $\frac{f^{2}(-q)}{f\left(-q^{3}\right)}$ & 0 & 14,17 \\
\hline
\end{tabular}

Also in [7], Garvan made the following stronger conjecture for the functions $f\left(-q^{6},-q^{10}\right) /\left(-q^{4} ; q^{4}\right)_{\infty}$ and $q f\left(-q^{2},-q^{14}\right) /\left(-q^{4} ; q^{4}\right)_{\infty}$ :

Conjecture 1.2. Define $b_{n}$ by

$$
\sum_{n=0}^{\infty} b_{n} q^{n}=\frac{f\left(-q^{6},-q^{10}\right)}{\left(-q^{4} ; q^{4}\right)_{\infty}}+q \frac{f\left(-q^{2},-q^{14}\right)}{\left(-q^{4} ; q^{4}\right)_{\infty}} .
$$




\section{Table 2}

\begin{tabular}{cccc}
\hline Function & $a$ & $\begin{array}{c}\text { Monotonic for } a n+b \\
\text { at least }\end{array}$ & $\begin{array}{c}\text { Strictly monotonic for } a n+b \\
\text { strictly greater than }\end{array}$ \\
\hline$\frac{f(-q) f\left(-q^{2}\right)}{f\left(-q^{4}\right)}$ & 4 & 149 & 169 \\
$\frac{f\left(-q^{2}\right) f\left(-q^{3}\right)}{f\left(-q^{6}\right)}$ & 6 & 550 & 580 \\
$\frac{f\left(-q^{6},-q^{10}\right)}{\left(-q^{4} ; q^{4}\right)_{\infty}}$ & 8 & 1262 & 1374 \\
$q \frac{f\left(-q^{2},-q^{14}\right)}{\left(-q^{4} ; q^{4}\right)_{\infty}}$ & 8 & 719 & 759 \\
$\frac{f^{2}(-q)}{f\left(-q^{3}\right)}$ & 3 & 95 & 95 \\
\hline
\end{tabular}

Then

$$
\begin{aligned}
(-1)^{n} b_{4 n} \geq 0 & \text { for all } n \geq 0, \\
(-1)^{n} b_{4 n+1} \geq 0 & \text { for all } n \geq 0, \\
(-1)^{n} b_{4 n+2} \geq 0 & \text { for all } n \geq 0, n \neq 3, \\
(-1)^{n+1} b_{4 n+3} \geq 0 & \text { for all } n \geq 0 .
\end{aligned}
$$

Furthermore, each of these subsequences is eventually monotonic.

Andrews and Lewis [3] made several conjectures regarding the signs of the coefficients of $f^{2}(-q) / f\left(-q^{3}\right)$ and $f(-q) f\left(-q^{2}\right) / f\left(-q^{4}\right)$. They showed that, if $M(r, m, n)$ is the number of partitions of $n$ with crank congruent to $r(\bmod m)$, then

$$
\begin{aligned}
\sum_{n=0}^{\infty}(M(0,3, n)-M(1,3, n)) q^{n} & =\frac{f^{2}(-q)}{f\left(-q^{3}\right)}, \\
\sum_{n=0}^{\infty}(M(0,4, n)-M(2,4, n)) q^{n} & =\frac{f(-q) f\left(-q^{2}\right)}{f\left(-q^{4}\right)} .
\end{aligned}
$$

They went on to make the following conjectures:

Conjecture 1.3. For all $n$, we have

$$
\begin{aligned}
M(0,3,3 n) & >M(1,3,3 n), \\
M(0,3,3 n+1) & <M(1,3,3 n+1), \\
M(0,3,3 n+2) & \leq M(1,3,3 n+2) \quad \text { if } n \neq 1 .
\end{aligned}
$$

Conjecture 1.4. For $n \neq 5$, we have

$$
\begin{array}{ll}
M(0,4, n) \geq M(2,4, n) & \text { if } n \equiv 0,3(\bmod 4), \\
M(0,4, n) \leq M(2,4, n) & \text { if } n \equiv 1,2(\bmod 4) .
\end{array}
$$


By (1.8) and (1.9), we easily see that Conjectures 1.3 and 1.4 are equivalent to the following.

CONJECTURE 1.5. Let $a_{1}(n)$ and $a_{5}(n)$ be the coefficients of $q^{n}$ in $f(-q) f\left(-q^{2}\right) / f\left(-q^{4}\right)$ and $f^{2}(-q) / f\left(-q^{3}\right)$ respectively. Then for $n \neq 5$, we have

$$
\begin{array}{ll}
a_{1}(n) \geq 0 & \text { if } n \equiv 0,3(\bmod 4), \\
a_{1}(n) \leq 0 & \text { if } n \equiv 1,2(\bmod 4), \\
a_{5}(n)>0 & \text { if } n \equiv 0(\bmod 3), \\
a_{5}(n)<0 & \text { if } n \equiv 1(\bmod 3), \\
a_{5}(n) \leq 0 & \text { if } n \equiv 2(\bmod 3) .
\end{array}
$$

D. M. Kane [10] recently found Conjecture 1.3 to be true by finding the following asymptotic formula for the coefficients of $f^{2}(-q) / f\left(-q^{3}\right)$.

Theorem 1.1. For all $n \geq 1$, we have

$$
a_{5}(n)=c_{5}(n) \frac{e^{\frac{\pi}{3} \sqrt{2(n-1 / 24) / 3}}}{\sqrt{6} \sqrt{n-1 / 24}}+E_{5}(n),
$$

where

$$
c_{5}(n)= \begin{cases}1.87 \ldots & \text { if } n \equiv 0(\bmod 3) \\ -1.53 \ldots & \text { if } n \equiv 1(\bmod 3) \\ -0.347 \ldots & \text { if } n \equiv 2(\bmod 3)\end{cases}
$$

and

$$
\left|E_{5}(n)\right| \leq 200(n-1 / 24)^{1 / 4}+2(n-1 / 24)^{1 / 4} e^{\frac{\pi}{6}} \sqrt{2(n-1 / 24) / 3} .
$$

The estimate (1.12) is given in the proof of Corollary 2 in [10]. We have corrected a misprint in the exponent of $n-1 / 24$ in the second term, and also made a trivial improvement in the first term, replacing the original $n^{1 / 4}$ by $(n-1 / 24)^{1 / 4}$. In this paper, we show that Conjectures $1.1,1.2$, and 1.5 are true.

\section{MAIN RESULTS}

We prove Conjectures 1.1, 1.2, and 1.5 by finding asymptotic formulas for the first four functions in (1.6). These formulas are summarized in the following theorem.

THEOREM 2.1. Let $a_{1}(n), a_{2}(n), a_{3}(n), a_{4}(n)$ be the coefficients of $q^{n}$ in the expansions of

$$
\frac{f(-q) f\left(-q^{2}\right)}{f\left(-q^{4}\right)}, \quad \frac{f\left(-q^{2}\right) f\left(-q^{3}\right)}{f\left(-q^{6}\right)}, \quad \frac{f\left(-q^{6},-q^{10}\right)}{\left(-q^{4} ; q^{4}\right)_{\infty}}, \quad q \frac{f\left(-q^{2},-q^{14}\right)}{\left(-q^{4} ; q^{4}\right)_{\infty}},
$$

respectively. Then 


$$
\begin{aligned}
a_{1}(n) & =c_{1}(n) \frac{e^{\frac{\pi}{4} \sqrt{2(n-1 / 24) / 3}}}{2 \sqrt{2(n-1 / 24)}}+E_{1}(n), \\
a_{2}(n) & =c_{2}(n) \frac{e^{\frac{\pi}{6} \sqrt{2(n-1 / 24) / 3}}}{2 \sqrt{3(n-1 / 24)}}+E_{2}(n), \\
a_{3}(2 n) & =(-1)^{n} c_{3}(2 n) \frac{e^{\frac{\pi}{8}} \sqrt{2(2 n-1 / 24) / 3}}{4 \sqrt{2 n-1 / 24}}+E_{3}(2 n),
\end{aligned}
$$

where $c_{1}(n), c_{2}(n)$, and $c_{3}(n)$ are approximately

$$
\begin{aligned}
& c_{1}(n)= \begin{cases}(-1)^{n / 2}(1.847759 \ldots) & \text { if } n \equiv 0(\bmod 2), \\
(-1)^{(n+1) / 2}(0.765366 \ldots) & \text { if } n \equiv 1(\bmod 2),\end{cases} \\
& c_{2}(n)= \begin{cases}(-1)^{n / 3}(1.9696155 \ldots) & \text { if } n \equiv 0(\bmod 3), \\
(-1)^{(n-1) / 3}(0.6840402 \ldots) & \text { if } n \equiv 1(\bmod 3), \\
(-1)^{(n-2) / 3}(-1.2855752 \ldots) & \text { if } n \equiv 2(\bmod 3),\end{cases} \\
& c_{3}(n)= \begin{cases}(-1)^{n / 4}(2.77407969 \ldots) & \text { if } n \equiv 0(\bmod 4), \\
(-1)^{(n-1) / 4}(2.3517512 \ldots) & \text { if } n \equiv 1(\bmod 4), \\
(-1)^{(n-2) / 4}(-0.5517987 \ldots) & \text { if } n \equiv 2(\bmod 4), \\
(-1)^{(n-3) / 4}(1.5713899 \ldots) & \text { if } n \equiv 3(\bmod 4),\end{cases}
\end{aligned}
$$

and

(2.10) $\left|E_{1}(n)\right| \leq 150(n-1 / 24)^{1 / 4}+0.71(n-1 / 24)^{1 / 4} e^{\frac{\pi}{8} \sqrt{2(n-1 / 24) / 3}}$,

(2.11) $\left|E_{2}(n)\right| \leq 1879(n-1 / 24)^{1 / 4}+0.468(n-1 / 24)^{1 / 4} e^{\frac{\pi}{12} \sqrt{2(n-1 / 24) / 3}}$,

(2.12) $\left|E_{3}(n)\right| \leq 68793(n-1 / 24)^{1 / 4}+0.1754(n-1 / 24)^{1 / 4} e^{\frac{\pi}{16} \sqrt{2(n-1 / 24) / 3}}$.

We can now easily derive the following corollary.

Corollary 2.1.1. Conjectures 1.1 and 1.2 are true. In particular, the subsequences $\left\{(-1)^{n} b_{4 n}\right\}_{n=0}^{\infty}$ and $\left\{(-1)^{n} b_{4 n+2}\right\}_{n=0}^{\infty}$ are strictly monotonic for $n \geq 362$, and the subsequences $\left\{(-1)^{n} b_{4 n+1}\right\}_{n=0}^{\infty}$ and $\left\{(-1)^{n} b_{4 n+3}\right\}_{n=0}^{\infty}$ are strictly monotonic for $n \geq 246$.

Proof. It is easy to see, from (1.12) and (2.10)-(2.12), that

$$
\left|E_{1}(n)\right| \leq E_{1}^{*}(n):= \begin{cases}300(n-1 / 24)^{1 / 4} & \text { if } n<278 \\ 1.42(n-1 / 24)^{1 / 4} e^{\frac{\pi}{8} \sqrt{2(n-1 / 24) / 3}} & \text { if } n \geq 278\end{cases}
$$




$$
\begin{aligned}
& \left|E_{2}(n)\right| \leq E_{2}^{*}(n):= \begin{cases}3758(n-1 / 24)^{1 / 4} & \text { if } n<1506, \\
0.936(n-1 / 24)^{1 / 4} e^{\frac{\pi}{12} \sqrt{2(n-1 / 24) / 3}} & \text { if } n \geq 1506,\end{cases} \\
& \left|E_{3}(n)\right| \leq E_{3}^{*}(n):= \begin{cases}137586(n-1 / 24)^{1 / 4} & \text { if } n<6454, \\
0.3508(n-1 / 24)^{1 / 4} e^{\frac{\pi}{16}} \sqrt{2(n-1 / 24) / 3} & \text { if } n \geq 6454,\end{cases} \\
& \left|E_{5}(n)\right| \leq E_{5}^{*}(n):= \begin{cases}400(n-1 / 24)^{1 / 4} & \text { if } n<117, \\
4(n-1 / 24)^{1 / 4} e^{\frac{\pi}{6}} \sqrt{2(n-1 / 24) / 3} & \text { if } n \geq 117 .\end{cases}
\end{aligned}
$$

Let us first consider Conjecture 1.1 for $a_{1}(n)$. Write $a_{1}(n)=f_{1}(n)+$ $E_{1}(n)$. It is clear from $(2.2)$ and $(2.7)$ that $f_{1}(n)$ and $f_{1}(n+4)$ have the same sign. Consider

$$
f_{1}^{\prime}(n)=c_{1}(n) \frac{e^{\frac{\pi}{4} \sqrt{2(n-1 / 24) / 3}}}{2 \sqrt{2}(n-1 / 24)}\left(\frac{\pi}{8} \sqrt{\frac{2}{3}}-\frac{1}{2 \sqrt{n-1 / 24}}\right) .
$$

Note that the expression in brackets is positive for $n>3$ and strictly increasing. Also, the fraction in front of the brackets is strictly increasing for $n>10$. Therefore, $\left|f_{1}(n) / c_{1}(n)\right|$ is concave up for $n>10$ and so we have

$$
(-1)^{\lfloor(n+1) / 2\rfloor}\left(a_{1}(n+4)-a_{1}(n)\right)>0
$$

whenever

$$
\left|4 f_{1}^{\prime}(n)\right|>\left|E_{1}^{*}(n+4)\right|+\left|E_{1}^{*}(n)\right|
$$

Since $\frac{\pi}{8} \sqrt{\frac{2}{3}}-\frac{1}{2 \sqrt{n-1 / 24}}>0.2$ when $n \geq 20$, and $\sqrt{x+a} \leq \sqrt{x}+\sqrt{a}$, we have, from (2.7),

$$
\left|4 f_{1}^{\prime}(n)\right| \geq 0.2259 \frac{e^{\frac{\pi}{4} \sqrt{2(n-1 / 24) / 3}}}{n-1 / 24}
$$

and

$$
\begin{aligned}
5.7(n-1 / 24)^{1 / 4} e^{\frac{\pi}{8} \sqrt{2(n-1 / 24) / 3}} & \geq 5.393(n+4-1 / 24)^{1 / 4} e^{\frac{\pi}{8} \sqrt{2(n-1 / 24) / 3}} \\
& \geq 2\left|E_{1}^{*}(n+4)\right|>\left|E_{1}^{*}(n+4)\right|+\left|E_{1}^{*}(n)\right|
\end{aligned}
$$

whenever $n \geq 278$. If $u=(\pi / 8) \sqrt{2(n-1 / 24) / 3}$, then $(2.13)$ would be implied by the condition

$$
e^{u}>433.44 u^{5 / 2}
$$

Note that if $u_{0} \geq 5 / 2$, then $e^{u_{0}}>C u_{0}^{5 / 2}$ implies $e^{u}>C u^{5 / 2}$ for all $u \geq u_{0}$. Thus it is easily verified that (2.14) is true for all $u \geq 12.5$. This corresponds to $n \geq 1520$.

Similarly, we let $a_{2}(n)=f_{2}(n)+E_{2}(n)$ and see that

$$
(-1)^{\lfloor(n+1) / 3\rfloor}\left(a_{2}(n+6)-a_{2}(n)\right)>0
$$

whenever

$$
\left|6 f_{2}^{\prime}(n)\right|>\left|E_{2}^{*}(n+6)\right|+\left|E_{2}^{*}(n)\right| .
$$


Using (2.3) and (2.8), and the fact that $\frac{\pi}{12} \sqrt{\frac{2}{3}}-\frac{1}{2 \sqrt{n-1 / 24}}>0.2137$ when $n \geq 200$, we find that

$$
\left|6 f_{2}^{\prime}(n)\right|>0.211366 \frac{e^{\frac{\pi}{8} \sqrt{2(n-1 / 24) / 3}}}{n-1 / 24} .
$$

We also find that, for $n \geq 1506$,

$$
3.6(n-1 / 24)^{1 / 4} e^{\frac{\pi}{12} \sqrt{2(n-1 / 24) / 3}} \geq 2\left|E_{2}^{*}(n+6)\right|>\left|E_{2}^{*}(n+6)\right|+\left|E_{2}^{*}(n)\right| .
$$

If $v=(\pi / 12) \sqrt{2(n-1 / 24) / 3}$, then $(2.15)$ is true whenever $n \geq 1506$ and

$$
e^{v}>806.232 v^{5 / 2} \text {. }
$$

This holds for $v \geq 13.2$, corresponding to $n \geq 3814$.

Now we let $a_{5}(n)=f_{5}(n)+E_{5}(n)$, and since $\lfloor(2 n+1) / 3\rfloor$ is even if and only if $n$ is divisible by 3 , we find that

$$
(-1)^{\lfloor(2 n+1) / 3\rfloor}\left(a_{5}(n+3)-a_{5}(n)\right)>0
$$

whenever

$$
\left|3 f_{5}^{\prime}(n)\right|>\left|E_{5}^{*}(n+3)\right|+\left|E_{5}^{*}(n)\right|
$$

We apply $(1.10)$ and $(1.11)$, and because $\frac{\pi}{6} \sqrt{\frac{2}{3}}-\frac{1}{2 \sqrt{n-1 / 24}}>0.315$ when $n \geq 20$, we deduce that

$$
\left|3 f_{5}^{\prime}(n)\right|>0.1341 \frac{e^{\frac{\pi}{3} \sqrt{2(n-1 / 24) / 3}}}{n-1 / 24} .
$$

We also find that, for $n \geq 117$,

$$
17.2(n-1 / 24)^{1 / 4} e^{\frac{\pi}{6} \sqrt{2(n-1 / 24) / 3}} \geq 2\left|E_{5}^{*}(n+3)\right|>\left|E_{5}^{*}(n+3)\right|+\left|E_{5}^{*}(n)\right| .
$$

If $w=(\pi / 6) \sqrt{2(n-1 / 24) / 3}$, then $(2.17)$ is true whenever $n \geq 117$ and

$$
e^{w}>1073.3 w^{5 / 2} \text {. }
$$

This holds whenever $w \geq 13.5$, corresponding to $n \geq 998$.

To prove Conjecture 1.1 for $a_{3}(n)$ and $a_{4}(n)$, we will prove Conjecture 1.2 with an explicit bound for monotonicity. Let, as in Conjecture $1.2, b_{2 n}:=$ $a_{3}(2 n)=f_{3}(2 n)+E_{3}(2 n), b_{2 n+1}:=a_{4}(2 n+1)=f_{3}(2 n+1)+E_{3}(2 n+1)$. By (2.4), (2.5), and (2.9) we see the $f_{3}(n)$ has the appropriate signs for Conjecture 1.2 to hold. Thus, we see that the subsequences (1.7) are increasing whenever

$$
\left|4 f_{3}^{\prime}(n)\right|>\left|E_{3}^{*}(n+4)\right|+\left|E_{3}^{*}(n)\right|
$$


Since $\frac{\pi}{16} \sqrt{\frac{2}{3}}-\frac{1}{2 \sqrt{n-1 / 24}}>0.1445$ when $n \geq 1000$, we find that

$$
\left|4 f_{3}^{\prime}(n)\right|> \begin{cases}0.092 \frac{e^{\frac{\pi}{8} \sqrt{2(n-1 / 24) / 3}}}{n-1 / 24} & \text { if } n \text { is even } \\ 0.2622 \frac{e^{\frac{\pi}{8} \sqrt{2(n-1 / 24) / 3}}}{n-1 / 24} & \text { if } n \text { is odd. }\end{cases}
$$

Also, since $(4 / n)^{1 / 4} \leq 0.1582$ when $n \geq 6400$, we have, for $n \geq 6454$,

$$
1.125(n-1 / 24)^{1 / 4} e^{\frac{\pi}{16} \sqrt{2(n-1 / 24) / 3}} \geq 2 E_{3}^{*}(n+4)>E_{3}^{*}(n+4)+E_{3}^{*}(n) .
$$

If $y=(\pi / 16) \sqrt{2(n-1 / 24) / 3}$, then (2.19) is true whenever $n \geq 6454$ and

$$
e^{y}> \begin{cases}1188.24 y^{5 / 2} & \text { if } n \text { is even, } \\ 416.93 y^{5 / 2} & \text { if } n \text { is odd }\end{cases}
$$

We easily check that (2.20) holds for $y \geq 13.7$ for $n$ even, corresponding to $n \geq 7303$, and $y \geq 12.5$ for $n$ odd, corresponding to $n \geq 6080$. It is now a simple matter to verify Conjectures 1.1 and 1.2 up to the values of $n$ we have computed here. We have carried out the computations in the computer algebra system PARI-GP version 2.0.20(beta) and found both conjectures to be true.

The following corollaries are now easily shown.

COROllary 2.1.2. Ramanujan's 10 tables are complete.

Proof. By Corollary 2.1.1, we need to check Ramanujan's tables up to the values of $n$ where the coefficients are strictly monotonic; after that, monotonicity ensures the coefficients are bounded away from 0,1 , and -1 . We have verified the tables in PARI-GP 2.0.20(beta) and found them to be complete.

Corollary 2.1.3. Conjecture 1.5 is true.

Proof. We have verified the conjecture up to $n=169$ using PARI-GP 2.0.20(beta). The monotonicity condition of Conjecture 1.1 guarantees the coefficients of $f(-q) f\left(-q^{2}\right) / f\left(-q^{4}\right)$ have the correct sign for $n>169$.

\section{THE METHOD OF PROOF}

We apply the circle method to prove Theorem 2.1. The approach will follow the method used by Rademacher (see Andrews [1, Chapter 5]) and the modification by Kane [10]. To obtain an estimate for the coefficient $a(n)$ of $s^{n}$ in the series expansion of a function $g(s)=\sum_{n=0}^{\infty} a(n) s^{n}$, we apply the 
residue theorem to find that

$$
a(n)=\frac{1}{2 \pi i} \oint_{|s|=r} \frac{g(s)}{s^{n+1}} d s
$$

where we have integrated along the positively oriented circle of radius $r$. We then apply a transformation formula to the integrand and evaluate a main term and estimate an error term. The details now follow.

3.1. Farey arcs and changes of variables. We integrate (3.1) over a circle of radius $r=e^{-2 \pi / N^{2}}=e^{-2 \pi \varrho}$ for some $N>0$ to be determined. We let $s=r e^{2 \pi i \theta}=e^{-2 \pi \varrho+2 \pi i \theta}$. Make the substitution $\theta=h / k+\varphi$ on each Farey arc centred at $e^{2 \pi i h / k}$, where $h / k$ is a Farey fraction of order $N$, and the endpoints of each arc are the mediants of consecutive Farey fractions of order $N$. That is, if $h / k$ and $h^{\prime} / k^{\prime}$ are a pair of consecutive Farey fractions of order $N$, then their mediant is defined to be the fraction $\left(h+h^{\prime}\right) /\left(k+k^{\prime}\right)$. Let $\xi_{h, k}=\left[-\theta_{h, k}^{\prime}, \theta_{h, k}^{\prime \prime}\right]$ represent the interval that $\varphi$ passes through on that arc, where $\theta_{h, k}^{\prime}, \theta_{h, k}^{\prime \prime}$ are the positive distances from $h / k$ to its neighbouring mediants. Then we have

$$
d s=2 \pi i e^{-2 \pi \varrho} e^{2 \pi i h / k} e^{2 \pi i \varphi} d \varphi
$$

so that

$$
a(n)=\sum_{\substack{0 \leq h \leq k \leq N \\(h, k)=1}} e^{-2 \pi i n h / k} \int_{\xi_{h, k}} g\left(e^{2 \pi i(h / k+i \varrho+\varphi)}\right) e^{2 \pi n \varrho} e^{-2 \pi i n \varphi} d \varphi .
$$

If we set $z=k(\varrho-i \varphi)$ and $\tau=(h+i z) / k$, then our integral becomes

$$
\begin{aligned}
a(n) & =\sum_{\substack{0 \leq h \leq k \leq N \\
(h, k)=1}} e^{-2 \pi i n h / k} \int_{\xi_{h, k}} g\left(e^{2 \pi i(h+i z) / k}\right) e^{2 \pi n \varrho} e^{-2 \pi i n \varphi} d \varphi \\
& =\sum_{\substack{0 \leq h \leq k \leq N \\
(h, k)=1}} e^{-2 \pi i n h / k} \int_{\xi_{h, k}} g\left(e^{2 \pi i \tau}\right) e^{2 \pi n \varrho} e^{-2 \pi i n \varphi} d \varphi .
\end{aligned}
$$

\subsection{The Dedekind eta-function and its transformation formula.}

It is useful to work with the theta function $f(a, b)$, defined in (1.4), in its product form, given by the famous Jacobi Triple Product Identity [6, Chapter 16, Entry 19],

$$
f(a, b)=(-a ; a b)_{\infty}(-b ; a b)_{\infty}(a b ; a b)_{\infty} .
$$

From (3.3) it easily follows that

$$
f(-q)=\left(q ; q^{3}\right)_{\infty}\left(q^{2} ; q^{3}\right)_{\infty}\left(q^{3} ; q^{3}\right)_{\infty}=(q ; q)_{\infty}
$$


We set $q=e^{2 \pi i \tau}$, with $\operatorname{Im}(\tau)>0$, and define the Dedekind eta-function by

$$
\eta(\tau):=q^{1 / 24}(q ; q)_{\infty}
$$

We use the following transformation formula of $\eta(\tau)$ to transform the quotients (2.1) on each of the Farey arcs. For a proof of the transformation formula, see [4, pp. 52-61].

Lemma 3.1. Let $\Gamma=\mathrm{SL}_{2}(\mathbb{Z})$. Then for $\gamma=\left(\begin{array}{ll}a & b \\ c & d\end{array}\right) \in \Gamma, c>0$, we have

$$
\eta(\gamma \tau)=e^{-\pi i s(d, c)} e^{\pi i(a+d) /(12 c)} \sqrt{-i(c \tau+d)} \eta(\tau),
$$

where $s(d, c)$ is the Dedekind sum given by

$$
s(d, c):=\sum_{n(\bmod c)}\left(\left(\frac{d n}{c}\right)\right)\left(\left(\frac{n}{c}\right)\right),
$$

with

$$
((x)):= \begin{cases}x-\lfloor x\rfloor-1 / 2 & \text { if } x \notin \mathbb{Z} \\ 0 & \text { if } x \in \mathbb{Z}\end{cases}
$$

and the square root is taken on the principal branch, with $z^{1 / 2}>0$ for $z>0$.

Setting

$$
F\left(e^{2 \pi i \tau}\right):=\frac{1}{(q ; q)_{\infty}}=\frac{1}{f(-q)}=\frac{e^{\pi i \tau / 12}}{\eta(\tau)}
$$

we use (3.6) to obtain the transformation formula

$$
F\left(e^{2 \pi i \tau}\right)=e^{\pi i(\tau-\gamma \tau) / 12} e^{-\pi i s(d, c)} e^{\pi i(a+d) /(12 c)} \sqrt{-i(c \tau+d)} F\left(e^{2 \pi i \gamma \tau}\right) .
$$

Since we use (3.9) on the Farey arcs, the $\gamma \in \Gamma$ we choose on each arc depends on the pair $(h, k)$, where $\operatorname{gcd}(h, k)=1$. Suppose we are given such a pair. Then for $(n, k)=1$, let $h_{n}=n h$ and choose an integer $h_{n}^{\prime}$ such that $h_{n}^{\prime} h_{n} \equiv-1(\bmod k)$, with $h_{n} h_{n}^{\prime}-b_{n} k=-1$. Note that $h_{1}=h$, and define $h_{1}^{\prime}=h^{\prime}$. Setting

$$
\gamma_{(n, k)}:=\left(\begin{array}{cc}
h_{n}^{\prime} & -b_{n} \\
k & -h_{n}
\end{array}\right) \in \Gamma
$$

and recalling that

$$
\tau=\frac{h+i z}{k},
$$

we obtain

$$
\begin{aligned}
\gamma_{(n, k)}(n \tau) & =\frac{h_{n}^{\prime} \frac{h_{n}+i n z}{k}-b_{n}}{k \frac{h_{n}+i n z}{k}-h_{n}}=\frac{h_{n}^{\prime} h_{n}-b_{n} k+i n h_{n}^{\prime} z}{k(i n z)} \\
& =\frac{h_{n}^{\prime}+i(n z)^{-1}}{k} .
\end{aligned}
$$


In particular, we have

$$
\operatorname{Im}\left(\gamma_{(n, k)}(n \tau)\right)=\frac{1}{k} \operatorname{Re}\left(\frac{1}{n z}\right) .
$$

Further, we also have, for $k=m l$,

$$
\operatorname{Im}\left(\gamma_{(n, l)}(m n \tau)\right)=\frac{1}{l} \operatorname{Re}\left(\frac{1}{n z}\right)=\frac{m}{k} \operatorname{Re}\left(\frac{1}{n z}\right) .
$$

Thus (3.9) yields, for $(n, k)=1$,

$$
\begin{aligned}
F\left(e^{2 \pi i n \tau}\right)= & e^{\frac{\pi i}{12}\left(\frac{h_{n}+i n z}{k}-\frac{h_{n}^{\prime}+i(n z)^{-1}}{k}\right)} e^{-\pi i s\left(-h_{n}, k\right)} e^{\pi i\left(h_{n}^{\prime}-h_{n}\right) /(12 k)} \\
& \times \sqrt{-i\left(k \frac{h_{n}+i n z}{k}-h_{n}\right)} F\left(e^{2 \pi i \gamma_{(n, k)}(n \tau)}\right) \\
= & e^{\frac{\pi}{12 k}\left(\frac{1}{n z}-n z\right)} e^{\pi i s\left(h_{n}, k\right)} \sqrt{n z} F\left(e^{2 \pi i \gamma_{(n, k)}(n \tau)}\right) .
\end{aligned}
$$

3.3. Bounds on $z, \varphi$, and other quantities. We use the following estimates in the course of the proof of Theorem 2.1. We begin with upper and lower bounds for the distance between a Farey fraction and its neighbouring mediants. For a proof, see a standard text in number theory, for example, Hardy and Wright [9, pp. 23-31].

In all that follows, we assume that $0 \leq h \leq k \leq N$ and $(h, k)=1$. So we have

$$
L_{1}:=\frac{1}{2 k N} \leq \theta_{h, k}^{\prime}, \theta_{h, k}^{\prime \prime} \leq \frac{1}{k N}=: L_{2} .
$$

This implies that

$$
|\varphi| \leq \frac{1}{k N}
$$

Therefore we have

$$
\begin{aligned}
\frac{2 \pi}{k} \operatorname{Re}\left(\frac{1}{m z}\right) & =\frac{2 \pi}{m k^{2}} \operatorname{Re}\left(\frac{1}{\varrho-i \varphi}\right)=\frac{2 \pi}{m k^{2}} \frac{\varrho}{\varrho^{2}+\varphi^{2}} \\
& \geq \frac{1}{m k^{2}} \frac{2 \pi N^{-2}}{N^{-4}+k^{-2} N^{-2}} \\
& =\frac{1}{m} \frac{2 \pi}{k^{2} N^{-2}+1} \geq \frac{1}{m} \frac{2 \pi}{1+1} \geq \frac{\pi}{m}
\end{aligned}
$$

since $k \leq N$. That is,

$$
\frac{2 \pi}{k} \operatorname{Re}\left(\frac{1}{m z}\right) \geq \frac{\pi}{m}
$$

We also have

$$
\operatorname{Im}(\tau)=\frac{1}{k} \operatorname{Re}(z)=\varrho=\frac{1}{N^{2}}>0
$$


and

$$
|z|^{-1 / 2}=\frac{1}{k^{1 / 2}} \frac{1}{\left(\varrho^{2}+\varphi^{2}\right)^{-1 / 4}} \leq \frac{1}{k^{1 / 2} \varrho^{1 / 2}}=k^{-1 / 2} N .
$$

Finally, we note that, for $x$ real, $0<x<1$, we have

$$
\begin{aligned}
\log (F(x)) & =\sum_{n=1}^{\infty}-\log \left(1-x^{n}\right)=\sum_{n=1}^{\infty} \sum_{m=1}^{\infty} \frac{x^{n m}}{m} \\
& =\sum_{n=1}^{\infty} x^{n} \sum_{d \mid n} \frac{1}{d} \leq \sum_{n=1}^{\infty} n x^{n}=\frac{x}{(1-x)^{2}} .
\end{aligned}
$$

So, we find that for any $y$ with $\operatorname{Im}(y)>0$,

$$
\begin{aligned}
\left|\frac{1}{F\left(e^{2 \pi i y}\right)}\right| & =\prod_{n=1}^{\infty}\left|\left(1-e^{2 \pi i n y}\right)\right| \leq \prod_{n=1}^{\infty}\left(1+\left|e^{2 \pi i n y}\right|\right) \\
& \leq \prod_{n=1}^{\infty} \frac{1}{1-\left|e^{2 \pi i n y}\right|}=F\left(\left|e^{2 \pi i y}\right|\right)=F\left(e^{-2 \pi \operatorname{Im}(y)}\right) \\
& \leq \exp \left(\frac{e^{-2 \pi \operatorname{Im}(y)}}{\left(1-e^{-2 \pi \operatorname{Im}(y)}\right)^{2}}\right)
\end{aligned}
$$

That is,

$$
\left|\frac{1}{F\left(e^{2 \pi i \tau}\right)}\right| \leq F\left(\left|e^{2 \pi i \tau}\right|\right) \leq \exp \left(\frac{e^{-2 \pi \operatorname{Im}(\tau)}}{\left(1-e^{-2 \pi \operatorname{Im}(\tau)}\right)^{2}}\right) .
$$

We also use the following trivial bound repeatedly:

$$
\sum_{k=1}^{N} k^{n} \leq \begin{cases}N^{n+1} & \text { if } n \geq 0 \\ \frac{1}{n+1} N^{n+1} & \text { if }-1<n<0\end{cases}
$$

3.4. Evaluating an integral. We need the following key lemma.

LemMa 3.2. Let $(h, k)=1$ and define

$$
I:=\int_{\xi_{h, k}} e^{\frac{\pi}{12 k}\left(\frac{1}{z}-z\right)} z^{-1 / 2} e^{2 \pi n \varrho} e^{-2 \pi i \varphi} d \varphi .
$$

Then

$$
I=\sqrt{\frac{2}{k(n-1 / 24)}} \cosh \left(\frac{\pi}{k} \sqrt{\frac{2}{3}(n-1 / 24)}\right)+E(I),
$$

where

$$
|E(I)| \leq 1.2828 \frac{e^{2 \pi(n-1 / 24) \varrho} N^{1 / 2}}{n-1 / 24} .
$$


Proof. First, we make a change of variable $w=z / k=\varrho-i \varphi$. So $i d w=$ $d \varphi$ and $-2 \pi i n \varphi=2 \pi n(w-\varrho)$. Then we have

$$
\begin{aligned}
I & =\int_{\varrho+i \theta_{h, k}^{\prime}}^{\varrho-i \theta_{h, k}^{\prime \prime}} e^{\frac{\pi}{12}} \frac{1}{k^{2} w} e^{-\frac{\pi}{12} w}(k w)^{-1 / 2} e^{2 \pi n \varrho} e^{2 \pi n(w-\varrho)} i d w \\
& =\frac{1}{2 \pi i} \int_{\varrho-i \theta_{h, k}^{\prime \prime}}^{\varrho+i \theta_{h, k}^{\prime}} 2 \pi e^{\frac{\pi}{12} \frac{1}{k^{2} w}} e^{-\frac{\pi}{12} w}(k w)^{-1 / 2} e^{2 \pi n w} d w .
\end{aligned}
$$

Since the absolute value of the integrand tends to 0 exponentially as $\operatorname{Re}(w)$ tends to $-\infty$, we may write $I$ as

$$
\begin{aligned}
I & =\frac{1}{2 \pi i}\left(\int_{-\infty}^{(0+)}-\int_{-\infty-i \theta_{h, k}^{\prime \prime}}^{\varrho-i \theta_{h, k}^{\prime \prime}}+\int_{-\infty+i \theta_{h, k}^{\prime}}^{\varrho+i \theta_{h, k}^{\prime}}\right) 2 \pi e^{\frac{\pi}{12} \frac{1}{k^{2} w}} \\
& =:(k w)^{-1 / 2} e^{2 \pi(n-1 / 24) w} d w \\
& J_{1}-J_{2}+J_{3},
\end{aligned}
$$

where $J_{1}$ is integrated around the contour starting from $-\infty-i \theta_{h, k}^{\prime \prime}$, and looping around the origin, oriented in the positive direction.

To bound $J_{2}$ and $J_{3}$, we note that on the lines $w=x+i \theta,-\infty<x \leq \varrho$, $\theta \in\left\{ \pm \theta_{h, k}^{\prime}, \pm \theta_{h, k}^{\prime \prime}\right\}$, we have

$$
\begin{aligned}
\frac{1}{2 k N} \leq|\theta| \leq \frac{1}{k N}, \quad\left|e^{2 \pi(n-1 / 24) w}\right| & \leq e^{2 \pi(n-1 / 24) x} \\
\operatorname{Re}\left(\frac{\pi}{12\left(k^{2}\right) w}\right) & =\frac{\pi}{12\left(k^{2}\right)} \frac{x}{x^{2}+\theta^{2}} \leq \frac{x \pi}{12\left(k^{2}\right) \theta^{2}} \\
& \leq \frac{\pi \varrho}{12\left(k^{2}\right)(2 k N)^{-2}} \leq \frac{\pi \varrho N^{2}}{3}=\frac{\pi}{3}
\end{aligned}
$$

and

$$
|w|^{-1 / 2} \leq\left|x^{2}+\theta^{2}\right|^{-1 / 4} \leq|\theta|^{-1 / 2} \leq \sqrt{2 k N} .
$$

So, for $j=2,3$, we find that

$$
\begin{aligned}
\left|J_{j}\right| & \leq \frac{1}{2 \pi} \int_{-\infty}^{\varrho} \frac{2 \pi}{\sqrt{k}} e^{\pi / 3} \sqrt{2 k N} e^{2 \pi(n-1 / 24) x} d x \\
& =\frac{e^{\pi / 3} \sqrt{2 N}}{2 \pi(n-1 / 24)} e^{2 \pi(n-1 / 24) \varrho} \leq 0.6414 \frac{e^{2 \pi(n-1 / 24) \varrho} N^{1 / 2}}{n-1 / 24} .
\end{aligned}
$$


To evaluate $J_{1}$, we $\operatorname{expand} \exp \left(\frac{\pi}{12 k^{2} w}\right)$ in a power series about the origin, so that

$$
J_{1}=\frac{1}{2 \pi i} \int_{-\infty}^{(0+)} \frac{2 \pi}{\sqrt{k}} w^{-1 / 2} e^{2 \pi(n-1 / 24) w} \sum_{m=0}^{\infty} \frac{\left(\frac{\pi}{12} \frac{1}{k^{2} w}\right)^{m}}{m !} d w .
$$

Now we make a change of variable $x=2 \pi(n-1 / 24) w$, and interchange the order of summation and integration (say, by the dominated convergence theorem). Thus,

$$
J_{1}=\frac{2 \pi}{\sqrt{k}} \sum_{m=0}^{\infty}\left(\frac{\pi}{12 k^{2}}\right)^{m}(2 \pi(n-1 / 24))^{m+1 / 2-1} \frac{1}{2 \pi i} \int_{-\infty}^{(0+)} \frac{e^{x} x^{-m-1 / 2}}{m !} d x
$$

To evaluate each integral, we use Hankel's loop integral formula [12, p. 245], which states that for $s \notin \mathbb{Z}$,

$$
\frac{1}{\Gamma(s)}=\frac{1}{2 \pi i} \int_{-\infty}^{(0+)} e^{z} z^{-s} d z
$$

Therefore,

$$
J_{1}=\frac{2 \pi}{\sqrt{k}} \sum_{m=0}^{\infty}\left(\frac{\pi^{2}(n-1 / 24)}{6 k^{2}}\right)^{m} \frac{1}{\sqrt{2 \pi(n-1 / 24)}} \frac{1}{m ! \Gamma(m+1 / 2)} .
$$

Applying the functional equation $\Gamma(n+1)=n \Gamma(n)$, and $\Gamma(1 / 2)=\sqrt{\pi}$, we find that

$$
\begin{aligned}
J_{1} & =\frac{2 \pi}{\sqrt{k}} \sum_{m=0}^{\infty}\left(\frac{\pi^{2}(n-1 / 24)}{6 k^{2}}\right)^{m} \frac{1}{\sqrt{2 \pi(n-1 / 24)}} \frac{2^{2 m}}{(2 m) ! \Gamma(1 / 2)} \\
& =\frac{\sqrt{2}}{\sqrt{k(n-1 / 24)}} \sum_{m=0}^{\infty}\left(\sqrt{\frac{4 \pi^{2}(n-1 / 24)}{6 k^{2}}}\right)^{2 m} \frac{1}{(2 m) !} \\
& =\sqrt{\frac{2}{k(n-1 / 24)}} \cosh \left(\frac{\pi}{k} \sqrt{\frac{2}{3}(n-1 / 24)}\right) .
\end{aligned}
$$

The lemma now follows from (3.23)-(3.25).

\section{PROOF OF THEOREM 2.1}

We now prove (2.2) in detail. The proofs of (2.3)-(2.5) are similar.

4.1. The quotient $\frac{f(-q) f\left(-q^{2}\right)}{f\left(-q^{4}\right)}$. We apply (3.2) with

$$
g\left(e^{2 \pi i \tau}\right)=\sum_{n=0}^{\infty} a_{1}(n) q^{n}=\frac{f(-q) f\left(-q^{2}\right)}{f\left(-q^{4}\right)}=\frac{F\left(e^{2 \pi i(4 \tau)}\right)}{F\left(e^{2 \pi i \tau}\right) F\left(e^{2 \pi i(2 \tau)}\right)} .
$$


Here $\tau$ is as in (3.10). We consider the three cases where $\operatorname{gcd}(k, 4)$ is 1,2 , or 4 , and transform $g$ accordingly.

CASE 1: $k$ is odd. Then we have, by (3.13),

$$
\begin{aligned}
\frac{F\left(e^{2 \pi i(4 \tau)}\right)}{F\left(e^{2 \pi i \tau}\right) F\left(e^{2 \pi i(2 \tau)}\right)}= & e^{\pi i\left(s\left(h_{4}, k\right)-s\left(h_{2}, k\right)-s(h, k)\right)} e^{\frac{\pi}{12 k}\left(\frac{1}{4 z}-\frac{1}{2 z}-\frac{1}{z}-4 z+2 z+z\right)} \\
& \times \frac{\sqrt{4 z}}{\sqrt{2 z} \sqrt{z}} \frac{F\left(e^{2 \pi i \gamma_{(4, k)}(4 \tau)}\right)}{F\left(e^{2 \pi i \gamma_{(1, k)}(\tau)}\right) F\left(e^{2 \pi i \gamma_{(2, k)}(2 \tau)}\right)} \\
= & e^{\pi i\left(s\left(h_{4}, k\right)-s\left(h_{2}, k\right)-s(h, k)\right)} \sqrt{2} e^{\frac{\pi}{12 k}\left(\frac{-5}{4 z}-z\right)} z^{-1 / 2} \\
& \times \frac{F\left(e^{2 \pi i \gamma_{(4, k)}(4 \tau)}\right)}{F\left(e^{2 \pi i \gamma_{(1, k)}(\tau)}\right) F\left(e^{2 \pi i \gamma_{(2, k)}(2 \tau)}\right)} .
\end{aligned}
$$

CASE $2: k \equiv 2(\bmod 4)$. We write $k=2 k^{\prime}$ with $k^{\prime}$ odd. Then we have

$$
4 \tau=\frac{4 h+4 i z}{2 k^{\prime}}, \quad \text { so that } 2 \tau=\frac{h+i z}{k^{\prime}} .
$$

Thus, applying (3.13), we find that

$$
\begin{aligned}
\frac{F\left(e^{2 \pi i(4 \tau)}\right)}{F\left(e^{2 \pi i \tau}\right) F\left(e^{2 \pi i(2 \tau)}\right)} & \\
= & e^{\pi i\left(s\left(h_{2}, k^{\prime}\right)-s\left(h, k^{\prime}\right)-s\left(h, 2 k^{\prime}\right)\right)} e^{\frac{\pi}{12 k^{\prime}}\left(\frac{1}{2 z}-\frac{1}{z}-2 z+z\right)-\frac{\pi}{12\left(2 k^{\prime}\right)}\left(\frac{1}{z}-z\right)} \\
& \quad \times \frac{\sqrt{2 z}}{\sqrt{z} \sqrt{z}} \frac{F\left(e^{2 \pi i \gamma_{\left(2, k^{\prime}\right)}(4 \tau)}\right)}{F\left(e^{2 \pi i \gamma_{\left(1,2 k^{\prime}\right)}(\tau)}\right) F\left(e^{2 \pi i \gamma_{\left(1, k^{\prime}\right)}(2 \tau)}\right)} \\
= & e^{\pi i\left(s\left(h_{2}, k^{\prime}\right)-s\left(h, k^{\prime}\right)-s\left(h, 2 k^{\prime}\right)\right)} \sqrt{2} e^{\frac{\pi}{12 k^{\prime}}\left(\frac{-1}{z}-\frac{z}{2}\right)} z^{-1 / 2} \\
& \times \frac{F\left(e^{2 \pi i \gamma_{\left(2, k^{\prime}\right)}(4 \tau)}\right)}{F\left(e^{2 \pi i \gamma_{\left(1,2 k^{\prime}\right)}(\tau)}\right) F\left(e^{2 \pi i \gamma_{\left(1, k^{\prime}\right)}(2 \tau)}\right)} .
\end{aligned}
$$

CASE $3: k \equiv 0(\bmod 4)$. We write $k=4 l$. We similarly obtain from $(3.13)$ :

$$
\begin{aligned}
& \frac{F\left(e^{2 \pi i(4 \tau)}\right)}{F\left(e^{2 \pi i \tau}\right) F\left(e^{2 \pi i(2 \tau)}\right)} \\
& =e^{\pi i(s(h, l)-s(h, 2 l)-s(h, 4 l))} e^{\frac{\pi}{12 k^{\prime \prime}}\left(\frac{1}{z}-z\right)-\frac{\pi}{12(2 l)}\left(\frac{1}{z}-z\right)-\frac{\pi}{12(4 l)}\left(\frac{1}{z}-z\right)} \\
& \quad \times \frac{\sqrt{z}}{\sqrt{z} \sqrt{z}} \frac{F\left(e^{2 \pi i \gamma_{(1, l)}(4 \tau)}\right)}{F\left(e^{2 \pi i \gamma_{(1,4 l)}(\tau)}\right) F\left(e^{2 \pi i \gamma_{(1,2 l)}(2 \tau)}\right)} \\
& =e^{\pi i(s(h, l)-s(h, 2 l)-s(h, 4 l))} e^{\frac{\pi}{12(4 l)}\left(\frac{1}{z}-z\right)} z^{-1 / 2} \\
& \quad \times \frac{F\left(e^{2 \pi i \gamma_{(1, l)}(4 \tau)}\right)}{F\left(e^{2 \pi i \gamma_{(1,4 l)}(\tau)}\right) F\left(e^{2 \pi i \gamma_{(1,2 l)}(2 \tau)}\right)} .
\end{aligned}
$$


We now subdivide our integral for $a_{1}(n)$ into 3 pieces, namely,

$$
\begin{aligned}
a_{1}(n)= & \left(\sum_{\substack{1 \leq k \leq N \\
k \\
\text { odd }}}+\sum_{\substack{1 \leq k \leq N \\
k \equiv 2(\bmod 4)}}+\sum_{\substack{1 \leq k \leq N \\
k \equiv 0(\bmod 4)}}\right) \sum_{\substack{0 \leq h \leq k \\
(h, k)=1}} e^{-2 \pi i n h / k} \\
& \times \int_{\xi_{h, k}} \frac{F\left(e^{2 \pi i(4 \tau)}\right)}{F\left(e^{2 \pi i \tau}\right) F\left(e^{2 \pi i(2 \tau)}\right)} e^{2 \pi n \varrho} e^{-2 \pi i n \varphi} d \varphi \\
=: & S_{1}(A)+S_{2}(A)+S_{3}(A),
\end{aligned}
$$

with

$$
A=\frac{F\left(e^{2 \pi i(4 \tau)}\right)}{F\left(e^{2 \pi i \tau}\right) F\left(e^{2 \pi i(2 \tau)}\right)} e^{2 \pi n \varrho} e^{-2 \pi i n \varphi} .
$$

For the values of $k$ in $S_{1}$, we apply the transformation formula (4.1) to $A$ so that

$$
\begin{aligned}
A= & e^{\pi i\left(s\left(h_{4}, k\right)-s\left(h_{2}, k\right)-s(h, k)\right)} \sqrt{2} e^{\frac{\pi}{12 k}\left(\frac{-5}{4 z}-z\right)} z^{-1 / 2} \\
& \times \frac{F\left(e^{2 \pi i \gamma_{(4, k)}(4 \tau)}\right)}{F\left(e^{2 \pi i \gamma_{(1, k)}(\tau)}\right) F\left(e^{2 \pi i \gamma_{(2, k)}(2 \tau)}\right)} e^{2 \pi n \varrho} e^{-2 \pi i n \varphi} .
\end{aligned}
$$

Using (3.11), (3.16), (3.18), and (3.19), we find that

$$
\begin{aligned}
|A| \leq & \sqrt{2} F\left(e^{-2 \pi(1 / k) \operatorname{Re}(1 /(4 z))}\right) F\left(e^{-2 \pi(1 / k) \operatorname{Re}(1 /(2 z))}\right) F\left(e^{-2 \pi(1 / k) \operatorname{Re}(1 / z)}\right) \\
& \times e^{-\frac{5(2 \pi)}{k} \operatorname{Re}\left(\frac{1}{96 z}\right)} e^{-\frac{2 \pi}{24 k} \operatorname{Re}(z)}|z|^{-1 / 2} e^{2 \pi n \varrho} \\
\leq & \sqrt{2} \exp \left(\frac{e^{-\pi / 4}}{\left(1-e^{-\pi / 4}\right)^{2}}+\frac{e^{-\pi / 2}}{\left(1-e^{-\pi / 2}\right)^{2}}+\frac{e^{-\pi}}{\left(1-e^{-\pi}\right)^{2}}\right) \\
& \times e^{-5 \pi / 96} e^{-2 \pi \varrho / 24} k^{-1 / 2} N e^{2 \pi n \varrho} \\
\leq & e^{1.756} e^{2 \pi(n-1 / 24) \varrho} k^{-1 / 2} N .
\end{aligned}
$$

By (3.14), the length of the interval $\xi_{h, k}$ is at most $2 / k N$. So we have

$$
\begin{aligned}
\left|S_{1}\right| & \leq \sum_{\substack{1 \leq k \leq N \\
k \text { odd }}} \sum_{\substack{\leq \leq h \leq k \\
\xi_{h, k}}} \int^{1.756} e^{2 \pi(n-1 / 24) \varrho} k^{-1 / 2} N d \varphi \\
& \leq \sum_{\substack{1 \leq k \leq N \\
k \text { odd }}} \sum_{1 \leq h \leq k} e^{1.756} e^{2 \pi(n-1 / 24) \varrho} \frac{2}{k^{3 / 2}} \\
& \leq 2 e^{1.756} e^{2 \pi(n-1 / 24) \varrho} \sum_{1 \leq k \leq N} k^{-1 / 2} \\
& \leq 4 e^{1.756} e^{2 \pi(n-1 / 24) \varrho} N^{1 / 2},
\end{aligned}
$$

where we have used (3.20) in the last inequality. 
Similarly, for values of $k$ in $S_{2}$, we apply (4.2) to obtain

$$
\begin{aligned}
A= & e^{\pi i\left(s\left(h_{2}, k^{\prime}\right)-s\left(h, k^{\prime}\right)-s\left(h, 2 k^{\prime}\right)\right)} \sqrt{2} e^{\frac{\pi}{12 k^{\prime}}\left(\frac{-1}{z}-\frac{z}{2}\right)} z^{-1 / 2} \\
& \times \frac{F\left(e^{2 \pi i \gamma_{\left(2, k^{\prime}\right)}(4 \tau)}\right)}{F\left(e^{2 \pi i \gamma_{\left(1,2 k^{\prime}\right)}(\tau)}\right) F\left(e^{2 \pi i \gamma_{\left(1, k^{\prime}\right)}(2 \tau)}\right)} e^{2 \pi n \varrho} e^{-2 \pi i n \varphi} .
\end{aligned}
$$

By (3.11), we have

$$
\operatorname{Im}\left(\gamma_{\left(n, k^{\prime}\right)}(2 n \tau)\right)=\frac{1}{k^{\prime}} \operatorname{Re}\left(\frac{1}{n z}\right)=\frac{2}{k} \operatorname{Re}\left(\frac{1}{n z}\right)
$$

Therefore, by (3.16), (3.18), and (3.19), we find that

$$
\begin{aligned}
|A| \leq & \sqrt{2} F\left(e^{-2 \pi(2 / k) \operatorname{Re}(1 /(2 z))}\right) F\left(e^{-2 \pi(2 / k) \operatorname{Re}(1 / z)}\right) F\left(e^{-2 \pi(1 / k) \operatorname{Re}(1 / z)}\right) \\
& \times e^{-\frac{2 \pi}{k} \operatorname{Re}\left(\frac{1}{12 z}\right)} e^{-\frac{2 \pi}{24 k} \operatorname{Re}(z)}|z|^{-1 / 2} e^{2 \pi n \varrho} \\
\leq & \sqrt{2} \exp \left(\frac{e^{-2 \pi / 2}}{\left(1-e^{-2 \pi / 2}\right)^{2}}+\frac{e^{-2 \pi}}{\left(1-e^{-2 \pi}\right)^{2}}+\frac{e^{-\pi}}{\left(1-e^{-\pi}\right)^{2}}\right) \\
& \times e^{-\pi / 12} \frac{1}{\sqrt{k \varrho}} e^{2 \pi(n+1 / 24) \varrho} \\
\leq & e^{-0.1655} e^{2 \pi(n-1 / 24) \varrho} k^{-1 / 2} N
\end{aligned}
$$

so that we have

$$
\begin{aligned}
\left|S_{2}\right| & \leq \sum_{\substack{1 \leq k \leq N \\
k \equiv 2(\bmod 4)}} \sum_{1 \leq h \leq k} 2 e^{-0.1655} e^{2 \pi(n-1 / 24) \varrho} k^{-3 / 2} \\
& \leq 4 e^{-0.1655} e^{2 \pi(n-1 / 24) \varrho} N^{1 / 2} .
\end{aligned}
$$

To estimate $S_{3}$, we rewrite it in the form

$$
S_{3}=\sum_{1 \leq 4 l \leq N} \sum_{\substack{0 \leq h \leq 4 l \\(h, 4 l)=1}} e^{-2 \pi i n h / 4 l} \int_{\xi_{h, 4 l}} \frac{F\left(e^{2 \pi i(4 \tau)}\right)}{F\left(e^{2 \pi i \tau}\right) F\left(e^{2 \pi i(2 \tau)}\right)} e^{2 \pi n \varrho} e^{-2 \pi i n \varphi} d \varphi
$$

where $\tau=(h+i z) / 4 l, z=(4 l)(\varrho-i \varphi)$, and recall that $k=4 l$. Define

$$
\begin{aligned}
\omega_{h, l} & :=e^{\pi i(s(h, l)-s(h, 2 l)-s(h, 4 l))}, \\
\tau^{\prime} & :=\gamma_{(1,4 l)}(\tau)=\frac{h^{\prime}+i z^{-1}}{4 l} .
\end{aligned}
$$

Note that, for $n=1,2,4$,

$$
\gamma_{(1,4 l / n)}(n \tau)=\frac{h^{\prime}+i z^{-1}}{l}=n \tau^{\prime}
$$


Apply the transformation formula (4.3) to $S_{3}$ so that

$$
\begin{aligned}
S_{3}= & \sum_{1 \leq 4 l \leq N} \sum_{\substack{0 \leq h \leq 4 l \\
(h, 4 l)=1}} e^{-2 \pi i n h / 4 l} \int_{\xi_{h, 4 l}} \omega_{h, l} e^{\frac{\pi}{12(4 l)}\left(\frac{1}{z}-z\right)} z^{-1 / 2} \\
& \times \frac{F\left(e^{2 \pi i \gamma_{(1, l)}(4 \tau)}\right)}{F\left(e^{2 \pi i \gamma_{(1,4 l)}(\tau)}\right) F\left(e^{2 \pi i \gamma(1,2 l)}(2 \tau)\right)} e^{2 \pi n \varrho} e^{-2 \pi i n \varphi} d \varphi \\
= & \sum_{1 \leq 4 l \leq N} \sum_{\substack{0 \leq h \leq 4 l \\
(h, 4 l)=1}} e^{-2 \pi i n h / 4 l} \int_{\xi_{h, 4 l}} \omega_{h, l} e^{\frac{\pi}{12(4 l)}\left(\frac{1}{z}-z\right)} z^{-1 / 2} \\
& \times \frac{F\left(e^{2 \pi i\left(4 \tau^{\prime}\right)}\right)}{F\left(e^{2 \pi i \tau^{\prime}}\right) F\left(e^{2 \pi i\left(2 \tau^{\prime}\right)}\right)} e^{2 \pi n \varrho} e^{-2 \pi i n \varphi} d \varphi \\
= & \sum_{1 \leq 4 l \leq N} \sum_{\substack{0 \leq h \leq 4 l \\
(h, 4 l)=1}} e^{-2 \pi i n h / 4 l} \\
& \times \int_{\xi_{h, 4 l}} \omega_{h, l} e^{\frac{\pi}{12(4 l)}\left(\frac{1}{z}-z\right)} z^{-1 / 2} e^{2 \pi n \varrho} e^{-2 \pi i n \varphi} d \varphi \\
& +\sum_{1 \leq 4 l \leq N} \sum_{\substack{0 \leq h \leq 4 l \\
(h, 4 l)=1}} e^{-2 \pi i n h / 4 l} \int \omega_{h, l} e^{\frac{\pi}{12(4 l)}\left(\frac{1}{z}-z\right)} z^{-1 / 2} \\
& \times\left(\frac{F\left(e^{2 \pi i\left(4 \tau^{\prime}\right)}\right)}{F\left(e^{2 \pi i \tau^{\prime}}\right) F\left(e^{2 \pi i\left(2 \tau^{\prime}\right)}\right)}-1\right) e^{2 \pi n \varrho} e^{-2 \pi i n \varphi} d \varphi \\
= & T_{1}+T_{2} . \quad
\end{aligned}
$$

The main term arises from $T_{1}$, and we give a bound for $T_{2}$. Note that

$$
\left|e^{2 \pi i \tau^{\prime}}\right|=e^{-\frac{2 \pi}{4 l} \operatorname{Re}(1 / z)},
$$

and

$$
\frac{F\left(q^{4}\right)}{F(q) F\left(q^{2}\right)}=\frac{(q ; q)_{\infty}\left(q^{2} ; q^{2}\right)_{\infty}}{\left(q^{4} ; q^{4}\right)_{\infty}}=(q ; q)_{\infty} \prod_{n=1}^{\infty}\left(1-q^{4 n-2}\right) .
$$

Thus $a_{1}(n)$ represents the number of partitions of $n$ into an even number of parts minus the number of partitions of $n$ into an odd number of parts, where each part is chosen from a restricted set (namely, the parts are distinct except possibly for those congruent to $2(\bmod 4)$, which may be repeated at most once). Thus, we have $\left|a_{1}(n)\right| \leq p(n)$, the total number of partitions of $n$, and so

$$
\begin{aligned}
\left|\left(\frac{F\left(e^{2 \pi i\left(4 \tau^{\prime}\right)}\right)}{F\left(e^{2 \pi i \tau^{\prime}}\right) F\left(e^{2 \pi i\left(2 \tau^{\prime}\right)}\right)}-1\right)\right| & \leq\left|\sum_{n=0}^{\infty} a_{n} e^{2 \pi i n \tau^{\prime}}-1\right| \leq \sum_{n=0}^{\infty} p(n)\left|e^{2 \pi i n \tau^{\prime}}\right|-1 \\
& =F\left(\left|e^{2 \pi i \tau^{\prime}}\right|\right)-1 .
\end{aligned}
$$


Therefore

$$
\begin{aligned}
\left|e^{\frac{\pi}{12(4 l)}\left(\frac{1}{z}\right)}\left(\frac{F\left(e^{2 \pi i\left(4 \tau^{\prime}\right)}\right)}{F\left(e^{2 \pi i \tau^{\prime}}\right) F\left(e^{2 \pi i\left(2 \tau^{\prime}\right)}\right)}-1\right)\right| & \leq e^{\frac{2 \pi}{4 l} \operatorname{Re}(1 / z) / 24}\left(F\left(e^{-\frac{2 \pi}{4 l} \operatorname{Re}(1 / z)}\right)-1\right) . \\
& =\frac{F(x)-1}{x^{1 / 24}}
\end{aligned}
$$

where $x=e^{-\frac{2 \pi}{4 l} \operatorname{Re}(1 / z)}$. Note that $x^{-1 / 24}(F(x)-1)$ is an increasing function in $x$. Now, by (3.16), $x \leq e^{-\pi}$, so by (3.19) we see that

$$
F(x) \leq \exp \left(\frac{e^{-\pi}}{\left(1-e^{-\pi}\right)^{2}}\right) \leq 1.049
$$

Thus,

$$
\begin{aligned}
\left|e^{\frac{\pi}{12(4 l)}\left(\frac{1}{z}\right)}\left(\frac{F\left(e^{2 \pi i\left(4 \tau^{\prime}\right)}\right)}{F\left(e^{2 \pi i \tau^{\prime}}\right) F\left(e^{2 \pi i\left(2 \tau^{\prime}\right)}\right)}-1\right)\right| & \leq \frac{F(x)-1}{x^{1 / 24}} \\
& \leq(1.049-1) e^{\pi / 24} \leq 0.0551 .
\end{aligned}
$$

So we have

$$
\begin{aligned}
\left|T_{2}\right| & \leq \sum_{1 \leq 4 l \leq N} \sum_{\substack{0 \leq h \leq 4 l \\
(h, 4 l)=1}} \int_{\substack{h, 4 l\\
}} 0.0551 e^{-\frac{\pi}{12(4 l)} \operatorname{Re}(z)}|z|^{-1 / 2} e^{2 \pi n \varrho} d \varphi \\
& \leq \sum_{1 \leq 4 l \leq N} \sum_{\substack{0 \leq h \leq 4 l \\
(h, 4 l)=1}} \int_{\substack{h, 4 l \\
(\leq 4 l \leq N}} 0.0551 e^{-2 \pi \varrho / 24}(4 l)^{-1 / 2} N e^{2 \pi n \varrho} d \varphi \\
& \leq \sum_{\substack{0 \leq h \leq 4 l \\
(h, 4 l)=1}} 0.0551 e^{2 \pi(n-1 / 24) \varrho} \frac{2}{(4 l)^{3 / 2}} \\
& \leq \sum_{1 \leq 4 l \leq N} 0.0551 e^{2 \pi(n-1 / 24) \varrho} \frac{2}{(4 l)^{1 / 2}} \leq 0.2204 e^{2 \pi(n-1 / 24) \varrho} N^{1 / 2} .
\end{aligned}
$$

We now turn our attention to calculating $T_{1}$. Applying (3.21) and (3.22) with $k=4 l$, we find that

$$
\text { (4.11) } \begin{aligned}
T_{1}= & \sum_{1 \leq 4 l \leq N} \sum_{\substack{0 \leq h \leq 4 l \\
(h, 4 l)=1}} e^{-2 \pi i n h / 4 l} \omega_{h, l} \\
& \times\left(\sqrt{\frac{2}{4 l(n-1 / 24)}} \cosh \left(\frac{\pi}{4 l} \sqrt{\frac{2}{3}(n-1 / 24)}\right)+E(I)\right) \\
= & D_{n}+\sum_{1 \leq 4 l \leq N} \sum_{\substack{0 \leq h \leq 4 l \\
(h, 4 l)=1}} \frac{e^{-2 \pi i n h / 4 l} \omega_{h, l}}{\sqrt{2 l(n-1 / 24)}} \cosh \left(\frac{\pi}{4 l} \sqrt{\frac{2}{3}(n-1 / 24)}\right)
\end{aligned}
$$


with

$$
\begin{aligned}
\left|D_{n}\right| & \leq \sum_{1 \leq 4 l \leq N} \sum_{\substack{0 \leq h \leq 4 l \\
(h, 4 l)=1}} 1.2828 \frac{e^{2 \pi(n-1 / 24) \varrho} N^{1 / 2}}{n-1 / 24} \\
& \leq 1.2828 \frac{e^{2 \pi(n-1 / 24) \varrho} N^{5 / 2}}{n-1 / 24} .
\end{aligned}
$$

By (4.4) and (4.9), and the estimates in (4.5), (4.6), (4.10), (4.11), and (4.12), we find that

$$
\begin{aligned}
a_{1}(n) & =S_{1}+S_{2}+S_{3}=S_{1}+S_{2}+T_{1}+T_{2} \\
& =\sum_{1 \leq 4 l \leq N} \sum_{\substack{0 \leq h \leq 4 l \\
(h, 4 l)=1}} \frac{e^{-2 \pi i n h / 4 l} \omega_{h, l}}{\sqrt{2 l(n-1 / 24)}} \cosh \left(\frac{\pi}{4 l} \sqrt{\frac{2}{3}(n-1 / 24)}\right)+G_{n}
\end{aligned}
$$

with

$$
\begin{aligned}
\left|G_{n}\right| \leq & 4 e^{1.756} e^{2 \pi(n-1 / 24) \varrho} N^{1 / 2}+4 e^{-0.1655} e^{2 \pi(n-1 / 24) \varrho} N^{1 / 2} \\
& +1.2828 e^{2 \pi(n-1 / 24) \varrho} \frac{N^{5 / 2}}{n-1 / 24}+0.2204 e^{2 \pi(n-1 / 24) \varrho} N^{1 / 2}
\end{aligned}
$$

Setting $N=\sqrt{2 \pi(n-1 / 24)}$, we find that $\varrho=\frac{1}{N^{2}}=\frac{1}{2 \pi(n-1 / 24)}$. Thus, for $n \geq 1$,

$$
\begin{aligned}
\left|G_{n}\right| & \leq e(2 \pi(n-1 / 24))^{1 / 4}\left(4 e^{1.756}+4 e^{-0.1655}+1.2828(2 \pi)+0.2204\right) \\
& \leq 150(n-1 / 24)^{1 / 4}
\end{aligned}
$$

In other words, for $n \geq 1$,

$$
\begin{aligned}
a_{1}(n)= & \sum_{1 \leq 4 l \leq N} \sum_{\substack{0 \leq h \leq 4 l \\
(h, 4 l)=1}} \frac{e^{-2 \pi i n h / 4 l} \omega_{h, l}}{\sqrt{2 l(n-1 / 24)}} \\
& \times \cosh \left(\frac{\pi}{4 l} \sqrt{\frac{2}{3}(n-1 / 24)}\right)+G_{n} .
\end{aligned}
$$

We estimate the main term with $l=1$, and find an upper bound for the remaining terms. So, for $n \geq 1$,

$$
\begin{aligned}
a_{1}(n)= & \frac{e^{-2 \pi i n / 4} \omega_{1,1}}{2 \sqrt{2(n-1 / 24)}} e^{\frac{\pi}{4} \sqrt{2(n-1 / 24) / 3}} \\
& +\frac{e^{-2 \pi i 3 n / 4} \omega_{3,1}}{2 \sqrt{2(n-1 / 24)}} e^{\frac{\pi}{4} \sqrt{2(n-1 / 24) / 3}}+H_{n} \\
= & \frac{e^{\frac{\pi}{4} \sqrt{2(n-1 / 24) / 3}}}{2 \sqrt{2(n-1 / 24)}}\left(i^{n} \omega_{1,1}+(-i)^{n} \omega_{3,1}\right)+H_{n}
\end{aligned}
$$


with

$$
\begin{aligned}
H_{n}= & G_{n}+\frac{e^{-2 \pi i n / 4} \omega_{1,1}}{2 \sqrt{2(n-1 / 24)}} e^{-\frac{\pi}{4} \sqrt{2(n-1 / 24) / 3}} \\
& +\frac{e^{-2 \pi i 3 n / 4} \omega_{3,1}}{2 \sqrt{2(n-1 / 24)}} e^{-\frac{\pi}{4} \sqrt{2(n-1 / 24) / 3}} \\
& +\sum_{2 \leq l \leq N / 4} \sum_{\substack{0 \leq h \leq 4 l \\
(h, 4 l)=1}} \frac{e^{-2 \pi i n h / 4 l} \omega_{h, l}}{2 \sqrt{2 l(n-1 / 24)}} \\
& \times\left(e^{\frac{\pi}{4 l} \sqrt{2(n-1 / 24) / 3}}+e^{-\frac{\pi}{4 l} \sqrt{2(n-1 / 24) / 3}}\right) .
\end{aligned}
$$

The terms with negative exponents are all $\leq 1$, and we bound the terms with positive exponents at $l=2$ to find that

$$
\begin{aligned}
\left|H_{n}\right| \leq & \left|G_{n}\right|+\frac{1}{\sqrt{2}} \\
& +\sum_{l=2}^{\lfloor N / 4\rfloor} \sum_{\substack{0 \leq h \leq 4 l \\
(h, 4 l)=1}} \frac{1}{2 \sqrt{2 l(n-1 / 24)}}\left(e^{\frac{\pi}{8} \sqrt{2(n-1 / 24) / 3}}+1\right) \\
\leq & \left|G_{n}\right|+\frac{1}{\sqrt{2}}+\sum_{l=2}^{\lfloor\sqrt{2 \pi(n-1 / 24)} / 4\rfloor} \frac{\sqrt{2 l}}{\sqrt{n-1 / 24}}\left(e^{\frac{\pi}{8} \sqrt{2(n-1 / 24) / 3}}+1\right) \\
\leq & \left|G_{n}\right|+\frac{1}{\sqrt{2}}+\frac{\sqrt{2}(\sqrt{2 \pi(n-1 / 24)} / 4)^{3 / 2}}{\sqrt{n-1 / 24}}\left(e^{\frac{\pi}{8} \sqrt{2(n-1 / 24) / 3}}+1\right) \\
\leq & 150(n-1 / 24)^{1 / 4}+0.71(n-1 / 24)^{1 / 4} e^{\frac{\pi}{8}} \sqrt{2(n-1 / 24) / 3}
\end{aligned}
$$

Here we have used (3.20) several times.

Finally, we use (3.7) and (3.8) to determine $i^{n} \omega_{1,1}+(-i)^{n} \omega_{3,1}$ explicitly. Recall

$$
\omega_{h, l}=e^{\pi i(s(h, l)-s(h, 2 l)-s(h, 4 l))}, \quad s(d, c)=\sum_{n(\bmod c)}\left(\left(\frac{d n}{c}\right)\right)\left(\left(\frac{n}{c}\right)\right),
$$

and

$$
((x))= \begin{cases}x-\lfloor x\rfloor-1 / 2 & \text { if } x \notin \mathbb{Z} \\ 0 & \text { if } x \in \mathbb{Z} .\end{cases}
$$

Thus, with a short computation, we find that

$$
\omega_{1,1}=e^{\pi i / 8}, \quad \omega_{3,1}=e^{-\pi i / 8} .
$$


So

$$
i^{n} \omega_{1,1}+(-i)^{n} \omega_{3,1}= \begin{cases}\sqrt{2+\sqrt{2}} & \text { if } n \equiv 0(\bmod 4), \\ -\sqrt{2-\sqrt{2}} & \text { if } n \equiv 1(\bmod 4), \\ -\sqrt{2+\sqrt{2}} & \text { if } n \equiv 2(\bmod 4), \\ \sqrt{2-\sqrt{2}} & \text { if } n \equiv 3(\bmod 4) .\end{cases}
$$

Equality (2.2) now follows from (4.14)-(4.16), with $H_{n}=E_{1}(n)$.

4.2. The quotient $\frac{f\left(-q^{2}\right) f\left(-q^{3}\right)}{f\left(-q^{6}\right)}$. The proof of $(2.3)$ is very similar to that of (2.2). In this case, we apply (3.2) with

$$
g\left(e^{2 \pi i \tau}\right)=\sum_{n=0}^{\infty} a_{2}(n) q^{n}=\frac{f\left(-q^{2}\right) f\left(-q^{3}\right)}{f\left(-q^{6}\right)}=\frac{F\left(e^{2 \pi i(6 \tau)}\right)}{F\left(e^{2 \pi i(2 \tau)}\right) F\left(e^{2 \pi i(3 \tau)}\right)},
$$

and we consider the cases where $\operatorname{gcd}(k, 6)$ is $1,2,3$, or 6 . The transformation formulas we obtain from (3.13) are listed below.

CASE $1: k \equiv 1,5(\bmod 6)$. We find that

$$
\begin{aligned}
\frac{F\left(e^{2 \pi i(6 \tau)}\right)}{F\left(e^{2 \pi i(3 \tau)}\right) F\left(e^{2 \pi i(2 \tau)}\right)}= & e^{\pi i\left(s\left(h_{6}, k\right)-s\left(h_{2}, k\right)-s\left(h_{3}, k\right)\right)} e^{\frac{\pi}{12 k}\left(\frac{-2}{3 z}-z\right)} z^{-1 / 2} \\
& \times \frac{F\left(e^{2 \pi i \gamma_{(6, k)}(6 \tau)}\right)}{F\left(e^{2 \pi i \gamma_{(3, k)}(3 \tau)}\right) F\left(e^{2 \pi i \gamma_{(2, k)}(2 \tau)}\right)} .
\end{aligned}
$$

CASE $2: k \equiv 2,4(\bmod 6)$, so that $k=2 k^{\prime},\left(k^{\prime}, 3\right)=1$. Then we have

$$
6 \tau=\frac{3 h+3 i z}{k^{\prime}}, \quad 3 \tau=\frac{3 h+3 i z}{k}, \quad 2 \tau=\frac{h+i z}{k^{\prime}} .
$$

Applying (3.13) we deduce that

$$
\begin{aligned}
\frac{F\left(e^{2 \pi i(6 \tau)}\right)}{F\left(e^{2 \pi i(3 \tau)}\right) F\left(e^{2 \pi i(2 \tau)}\right)}= & e^{\pi i\left(s\left(h_{3}, k^{\prime}\right)-s\left(h, k^{\prime}\right)-s\left(h_{3}, 2 k^{\prime}\right)\right)} e^{\frac{\pi}{12 k^{\prime}}\left(\frac{-5}{6 z}-\frac{z}{2}\right)} z^{-1 / 2} \\
& \times \frac{F\left(e^{2 \pi i \gamma_{\left(3, k^{\prime}\right)}(6 \tau)}\right)}{F\left(e^{2 \pi i \gamma_{\left(3,2 k^{\prime}\right)}(3 \tau)}\right) F\left(e^{2 \pi i \gamma_{\left(1, k^{\prime}\right)}(2 \tau)}\right)} .
\end{aligned}
$$

CASE $3: k \equiv 3(\bmod 6)$, so that $k=3 k^{\prime \prime}, k^{\prime \prime}$ odd. Then

$$
6 \tau=\frac{2 h+2 i z}{k^{\prime \prime}}, \quad 3 \tau=\frac{h+i z}{k^{\prime \prime}}, \quad 2 \tau=\frac{2 h+2 i z}{k} .
$$

Therefore, we find that

$$
\begin{aligned}
\frac{F\left(e^{2 \pi i(6 \tau)}\right)}{F\left(e^{2 \pi i(3 \tau)}\right) F\left(e^{2 \pi i(2 \tau)}\right)}= & e^{\pi i\left(s\left(h_{2}, k^{\prime \prime}\right)-s\left(h, k^{\prime \prime}\right)-s\left(h_{2}, 3 k^{\prime \prime}\right)\right)} e^{\frac{\pi}{12 k^{\prime \prime}}\left(\frac{-2}{3 z}-\frac{z}{3}\right)} z^{-1 / 2} \\
& \times \frac{F\left(e^{2 \pi i \gamma_{\left(2, k^{\prime \prime}\right)}(6 \tau)}\right)}{F\left(e^{2 \pi i \gamma_{\left(1, k^{\prime \prime}\right)}(3 \tau)}\right) F\left(e^{2 \pi i \gamma_{\left(2,3 k^{\prime \prime}\right)}(2 \tau)}\right)} .
\end{aligned}
$$


CASE $4: k \equiv 0(\bmod 6)$, so $k=6 l$. We have

$$
6 \tau=\frac{h+i z}{l}, \quad 3 \tau=\frac{h+i z}{2 l}, \quad 2 \tau=\frac{h+i z}{3 l} .
$$

Thus,

$$
\begin{aligned}
\frac{F\left(e^{2 \pi i(6 \tau)}\right)}{F\left(e^{2 \pi i(3 \tau)}\right) F\left(e^{2 \pi i(2 \tau)}\right)}= & e^{\pi i(s(h, l)-s(h, 2 l)-s(h, 3 l))} e^{\frac{\pi}{12(6 l)}\left(\frac{1}{z}-z\right)} z^{-1 / 2} \\
& \times \frac{F\left(e^{2 \pi i \gamma_{(1, l)}(6 \tau)}\right)}{F\left(e^{2 \pi i \gamma_{(1,2 l)}(3 \tau)}\right) F\left(e^{2 \pi i \gamma_{(1,3 l)}(2 \tau)}\right)}
\end{aligned}
$$

We subdivide our integral for $a_{2}(n)$ into four pieces, corresponding to each of these four cases, to obtain

$$
\begin{aligned}
a_{2}(n)= & \sum_{\substack{1 \leq k \leq N \\
k \equiv \pm 1(\bmod 6)}}+\sum_{\substack{1 \leq k \leq N \\
k \equiv \pm 2(\bmod 6)}}+\sum_{\substack{1 \leq k \leq N \\
k \equiv 3(\bmod 6)}}+\sum_{\substack{1 \leq k \leq N \\
k \equiv 0(\bmod 6)}} e^{-2 \pi i n h / k} \\
& \times \sum_{0 \leq h \leq k,(h, k)=1} \sum_{\xi_{h, k}} \frac{F\left(e^{2 \pi i(6 \tau)}\right)}{F\left(e^{2 \pi i(3 \tau)}\right) F\left(e^{2 \pi i(2 \tau)}\right)} e^{2 \pi n \varrho} e^{-2 \pi i n \varphi} d \varphi \\
= & : S_{1}\left(A_{1}\right)+S_{2}\left(A_{2}\right)+S_{3}\left(A_{3}\right)+S_{4}\left(A_{4}\right),
\end{aligned}
$$

where

$$
A_{1}=A_{2}=A_{3}=A_{4}=\frac{F\left(e^{2 \pi i(6 \tau)}\right)}{F\left(e^{2 \pi i(3 \tau)}\right) F\left(e^{2 \pi i(2 \tau)}\right)} e^{2 \pi n \varrho} e^{-2 \pi i n \varphi} .
$$

Transform $A_{1}$ with (4.17), $A_{2}$ with (4.18), and $A_{3}$ with (4.19), and apply (3.11), (3.12), (3.16), (3.18), and (3.19) to each to find that

$$
\begin{aligned}
\left|A_{1}\right| & \leq e^{\frac{\pi}{12 k} \operatorname{Re}\left(\frac{-2}{3 z}-z\right)}|z|^{-1 / 2}\left|\frac{F\left(e^{-\frac{2 \pi}{k} \operatorname{Re}\left(\frac{1}{6 z}\right)}\right)}{F\left(e^{-\frac{2 \pi}{k} \operatorname{Re}\left(\frac{1}{3 z}\right)}\right) F\left(e^{-\frac{2 \pi}{k} \operatorname{Re}\left(\frac{1}{2 z}\right)}\right)}\right| e^{2 \pi n \varrho} \\
& \leq e^{4.6424} e^{2 \pi(n-1 / 24) \varrho} k^{-1 / 2} N, \\
\left|A_{2}\right| & \leq e^{\frac{\pi}{12 k^{\prime}} \operatorname{Re}\left(\frac{-5}{6 z}-\frac{z}{2}\right)}|z|^{-1 / 2}\left|\frac{F\left(e^{-2 \pi \frac{2}{k} \operatorname{Re}\left(\frac{1}{3 z}\right)}\right)}{F\left(e^{-\frac{2 \pi}{k} \operatorname{Re}\left(\frac{1}{3 z}\right)}\right) F\left(e^{-2 \pi \frac{2}{k} \operatorname{Re}\left(\frac{1}{z}\right)}\right)}\right| e^{2 \pi n \varrho} \\
& \leq e^{0.776806} e^{2 \pi(n-1 / 24) \varrho} k^{-1 / 2} N, \quad\left|\frac{F\left(e^{-2 \pi \frac{3}{k} \operatorname{Re}\left(\frac{1}{2 z}\right)}\right)}{F\left(e^{-2 \pi \frac{3}{k} \operatorname{Re}\left(\frac{1}{z}\right)}\right) F\left(e^{-\frac{2 \pi}{k} \operatorname{Re}\left(\frac{1}{2 z}\right)}\right)}\right| e^{2 \pi n \varrho} \\
\left|A_{3}\right| & \leq e^{\frac{\pi}{12 k^{\prime \prime}} \operatorname{Re}\left(\frac{-2}{3 z}-\frac{z}{3}\right)}|z|^{-1 / 2} \mid \frac{}{} \\
& \leq e^{0.0787343} e^{2 \pi(n-1 / 24) \varrho} k^{-1 / 2} N .
\end{aligned}
$$


By (3.14), the interval $\xi_{h, k}$ has length at most $2 / k N$. Therefore,

$$
\begin{aligned}
& \left|S_{1}\right|+\left|S_{2}\right|+\left|S_{3}\right| \\
& \leq \sum_{\substack{1 \leq k \leq N \\
k \equiv \pm 1(\bmod 6)}} \sum_{\substack{0 \leq h \leq k,(h, k)=1\\
}} 2 e^{4.6424} e^{2 \pi(n-1 / 24) \varrho} k^{-3 / 2}
\end{aligned}
$$

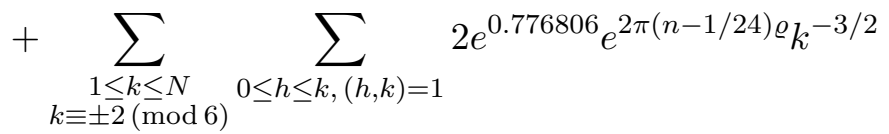

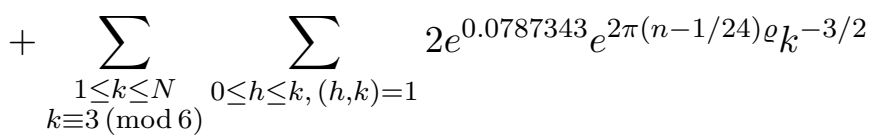

$$
\begin{aligned}
& \leq 2\left(e^{4.6424}+e^{0.776806}+e^{0.0787343}\right) e^{2 \pi(n-1 / 24) \varrho} \sum_{1 \leq k \leq N} k^{-1 / 2} \\
& \leq 4\left(e^{4.6424}+e^{0.776806}+e^{0.0787343}\right) e^{2 \pi(n-1 / 24) \varrho} \sqrt{N}
\end{aligned}
$$

Here we have used (3.20) in the last inequality.

To estimate $S_{4}$, rewrite it with $k=6 l$, so that we have $\tau=(h+i z) / 6 l$ and $z=(6 l)(\varrho-i \varphi)$. Therefore,

$$
S_{4}=\sum_{1 \leq 6 l \leq N} \sum_{\substack{1 \leq h \leq 6 l \\(h, 6 l)=1}} e^{-2 \pi i n h / 6 l} \int_{\xi_{h, 6 l}} \frac{F\left(e^{2 \pi i(6 \tau)}\right)}{F\left(e^{2 \pi i(3 \tau)}\right) F\left(e^{2 \pi i(2 \tau)}\right)} e^{2 \pi n \varrho} e^{-2 \pi i n \varphi} d \varphi .
$$

Applying the transformation formula (4.20) and setting

$$
\omega_{h, l}^{\prime}:=e^{\pi i(s(h, l)-s(h, 2 l)-s(h, 3 l))},
$$

we find that

$$
\begin{aligned}
S_{4}= & \sum_{1 \leq 6 l \leq N} \sum_{\substack{1 \leq h \leq 6 l \\
(h, 6 l)=1}} e^{-2 \pi i n h / 6 l} \int_{\xi_{h, 6 l}} \omega_{h, l}^{\prime} e^{\frac{\pi}{12(6 l)}\left(\frac{1}{z}-z\right)} z^{-1 / 2} \\
& \times \frac{F\left(e^{2 \pi i \gamma_{(1, l)}(6 \tau)}\right)}{F\left(e^{2 \pi i \gamma_{(1,2 l)}(3 \tau)}\right) F\left(e^{2 \pi i \gamma_{(1,3 l)}(2 \tau)}\right)} e^{2 \pi n \varrho} e^{-2 \pi i n \varphi} d \varphi .
\end{aligned}
$$

We define

$$
\tau^{\prime}:=\frac{h^{\prime}+i z^{-1}}{6 l}
$$

so that for $n=1,2,3,6$,

$$
\gamma_{(1,6 l / n)}(n \tau)=n \tau^{\prime}
$$

Thus, 


$$
\begin{aligned}
S_{4}= & \sum_{1 \leq 6 l \leq N} \sum_{\substack{1 \leq h \leq 6 l \\
(h, 6 l)=1}} e^{-2 \pi i n h / 6 l} \\
& \times \int_{\xi_{h, 6 l}} \omega_{h, l}^{\prime} e^{\frac{\pi}{12(6 l)}\left(\frac{1}{z}-z\right)} z^{-1 / 2} e^{2 \pi n \varrho} e^{-2 \pi i n \varphi} d \varphi \\
& +\sum_{1 \leq 6 l \leq N} \sum_{\substack{(h, 6 l)=1 \\
(h \leq 6 l}} e^{-2 \pi i n h / 6 l} \int_{\xi_{h, 6 l}} \omega_{h, l}^{\prime} e^{\frac{\pi}{12(6 l)}\left(\frac{1}{z}-z\right)} z^{-1 / 2} \\
& \times\left(\frac{F\left(e^{2 \pi i\left(6 \tau^{\prime}\right)}\right)}{F\left(e^{2 \pi i\left(3 \tau^{\prime}\right)}\right) F\left(e^{2 \pi i\left(2 \tau^{\prime}\right)}\right)}-1\right) e^{2 \pi n \varrho} e^{-2 \pi i n \varphi} d \varphi \\
= & : T_{1}+T_{2} .
\end{aligned}
$$

We estimate $\left|T_{2}\right|$ by noting that

$$
\frac{f\left(-q^{2}\right) f\left(-q^{3}\right)}{f\left(-q^{6}\right)}=\left(q^{2} ; q^{2}\right)_{\infty}\left(q^{3} ; q^{6}\right)_{\infty}
$$

So $\left|a_{2}(n)\right| \leq p(n)$, since it represents the number of partitions of $n$ into an even number of parts, minus the number of partitions of $n$ into an odd number of parts, where the parts are distinct and either even or congruent to $3(\bmod 6)$. Therefore,

$$
\begin{gathered}
\left|e^{\frac{\pi}{12(6 l)}\left(\frac{1}{z}\right)}\left(\frac{F\left(e^{2 \pi i\left(6 \tau^{\prime}\right)}\right)}{F\left(e^{2 \pi i\left(3 \tau^{\prime}\right)}\right) F\left(e^{2 \pi i\left(2 \tau^{\prime}\right)}\right)}-1\right)\right| \\
\leq e^{\frac{2 \pi}{6 l} \operatorname{Re}\left(\frac{1}{z}\right) / 24}\left(F\left(e^{\frac{-2 \pi}{6 l} \operatorname{Re}\left(\frac{1}{z}\right)}\right)-1\right) \\
\leq e^{\pi / 24}\left(\exp \left(\frac{e^{-\pi}}{\left(1-e^{-\pi}\right)^{2}}\right)-1\right) \leq 0.0551 .
\end{gathered}
$$

Thus, applying (3.14), (3.18), and (3.20), we have

$$
\begin{aligned}
\left|T_{2}\right| & \leq \sum_{1 \leq 6 l \leq N} \sum_{\substack{1 \leq h \leq 6 l \\
(h, 6 l)=1}} \int_{\xi_{h, 6 l}} 0.0551 e^{-(2 \pi / 24)(1 / 6 l) \operatorname{Re}(z)}|z|^{-1 / 2} e^{2 \pi n \varrho} d \varphi \\
& \leq \sum_{1 \leq 6 l \leq N} \sum_{\substack{1 \leq h \leq 6 l \\
(h, 6 l)=1}} \int_{\xi_{h, 6 l}} 0.0551 e^{2 \pi(n-1 / 24) \varrho} k^{-1 / 2} N d \varphi \\
& \leq \sum_{1 \leq 6 l \leq N} \sum_{\substack{1 \leq h \leq 6 l \\
(h, 6 l)=1}} 0.1102 e^{2 \pi(n-1 / 24) \varrho} k^{-3 / 2} \\
& \leq 0.2204 e^{2 \pi(n-1 / 24) \varrho \sqrt{N}} .
\end{aligned}
$$


To calculate $T_{1}$, we apply (3.21) and (3.22) with $k=6 l$, so that

$$
\begin{aligned}
T_{1}= & \sum_{1 \leq 6 l \leq N} \sum_{\substack{1 \leq h \leq 6 l \\
(h, 6 l)=1}} e^{-2 \pi i n h / 6 l} \omega_{h, l}^{\prime} \\
& \times\left(\sqrt{\frac{2}{6 l(n-1 / 24)}} \cosh \left(\frac{\pi}{6 l} \sqrt{\frac{2}{3}(n-1 / 24)}\right)+E(I)\right) \\
= & D_{n}+\sum_{1 \leq 6 l \leq N} \sum_{\substack{1 \leq h \leq 6 l \\
(h, 6 l)=1}} \frac{e^{-2 \pi i n h / 6 l} \omega_{h, l}^{\prime}}{\sqrt{3 l(n-1 / 24)}} \cosh \left(\frac{\pi}{6 l} \sqrt{\frac{2}{3}(n-1 / 24)}\right),
\end{aligned}
$$

where

$$
\begin{aligned}
\left|D_{n}\right| & \leq \sum_{1 \leq 6 l \leq N} \sum_{\substack{1 \leq h \leq 6 l \\
(h, 6 l)=1}} 1.2828 e^{2 \pi(n-1 / 24) \varrho} N^{1 / 2}(n-1 / 24)^{-1} \\
& \leq 1.2828 \frac{e^{2 \pi(n-1 / 24) \varrho} N^{5 / 2}}{n-1 / 24} .
\end{aligned}
$$

Combining (4.21), (4.27), and the estimates in (4.25), (4.28), (4.29), and (4.30), we obtain

$$
\begin{aligned}
a_{2}(n) & =S_{1}+S_{2}+S_{3}+S_{4}=S_{1}+S_{2}+S_{3}+T_{1}+T_{2} \\
& =\sum_{1 \leq 6 l \leq N} \sum_{\substack{1 \leq h \leq 6 l \\
(h, 6 l)=1}} \frac{e^{-2 \pi i n h / 6 l} \omega_{h, l}^{\prime}}{\sqrt{3 l(n-1 / 24)}} \cosh \left(\frac{\pi}{6 l} \sqrt{\frac{2}{3}(n-1 / 24)}\right)+G_{n},
\end{aligned}
$$

where

$$
\begin{aligned}
\left|G_{n}\right| \leq & \left|S_{1}\right|+\left|S_{2}\right|+\left|S_{3}\right|+\left|T_{2}\right|+\left|D_{n}\right| \\
\leq & 4\left(e^{4.6424}+e^{0.776806}+e^{0.0787343}\right) e^{2 \pi(n-1 / 24) \varrho} \sqrt{N} \\
& +0.2204 e^{2 \pi(n-1 / 24) \varrho} \sqrt{N}+1.2828 e^{2 \pi(n-1 / 24) \varrho} N^{5 / 2}(n-1 / 24)^{-1} .
\end{aligned}
$$

Set $N=\sqrt{2 \pi(n-1 / 24)}$, so $\varrho=N^{-2}=\frac{1}{2 \pi(n-1 / 24)}$. Therefore,

$$
\begin{aligned}
\left|G_{n}\right| \leq & e(2 \pi)^{1 / 4}\left\{4 e^{4.6424}+4 e^{0.777}+4 e^{0.08}+0.2204+(2 \pi) 1.2828\right\} \\
& \times(n-1 / 24)^{1 / 4} \\
\leq & 1878.5(n-1 / 24)^{1 / 4} .
\end{aligned}
$$

We now estimate the main term using $l=1$ and bound the remainder of the sum. We find that, for $n \geq 1$,

$$
a_{2}(n)=\frac{e^{-2 \pi i n / 6} \omega_{1,1}^{\prime}+e^{-2 \pi i n 5 / 6} \omega_{5,1}^{\prime}}{2 \sqrt{3(n-1 / 24)}} e^{\frac{\pi}{6} \sqrt{2(n-1 / 24) / 3}}+H_{n}
$$


with

$$
\begin{aligned}
\left|H_{n}\right| \leq & \left|G_{n}\right|+\frac{1}{\sqrt{3(n-1 / 24)}} e^{-\frac{\pi}{6} \sqrt{2(n-1 / 24) / 3}} \\
& +\sum_{2 \leq l \leq N / 6} \sum_{\substack{1 \leq h \leq 6 l \\
(h, 6 l)=1}} \frac{1}{\sqrt{3 l(n-1 / 24)}} \frac{e^{\frac{\pi}{6 l} \sqrt{2(n-1 / 24) / 3}}+e^{-\frac{\pi}{6 l} \sqrt{2(n-1 / 24) / 3}}}{2} .
\end{aligned}
$$

We apply $(3.7)$ and $(3.8)$ to compute $e^{-2 \pi i n / 6} \omega_{1,1}^{\prime}+e^{-2 \pi i n 5 / 6} \omega_{5,1}^{\prime}$. Recall

$$
\omega_{h, l}^{\prime}=e^{\pi i(s(h, l)-s(h, 2 l)-s(h, 3 l))},
$$

and so

$$
\omega_{1,1}^{\prime}=e^{-\pi i / 18}, \quad \omega_{5,1}^{\prime}=e^{\pi i / 18}
$$

Therefore,

$$
e^{-2 \pi i n / 6} \omega_{1,1}^{\prime}+e^{-2 \pi i n 5 / 6} \omega_{5,1}^{\prime}= \begin{cases}2 \cos (\pi / 18) & \text { if } n \equiv 0(\bmod 6) \\ 2 \cos (7 \pi / 18) & \text { if } n \equiv 1(\bmod 6) \\ 2 \cos (13 \pi / 18) & \text { if } n \equiv 2(\bmod 6) \\ 2 \cos (19 \pi / 18) & \text { if } n \equiv 3(\bmod 6) \\ 2 \cos (11 \pi / 18) & \text { if } n \equiv 4(\bmod 6) \\ 2 \cos (5 \pi / 18) & \text { if } n \equiv 5(\bmod 6)\end{cases}
$$

To find an upper estimate for $\left|H_{n}\right|$, we bound all the terms involving positive exponents using $l=2$ and those involving negative exponents with $l=1$, so that

$$
\begin{aligned}
\left|H_{n}\right| \leq & \left|G_{n}\right|+\frac{1}{\sqrt{3(n-1 / 24)}} \\
& +\sum_{2 \leq l \leq N / 6} \sum_{\substack{1 \leq h \leq 6 l \\
(h, 6 l)=1}} \frac{1}{\sqrt{3 l(n-1 / 24)}} \frac{e^{\frac{\pi}{12} \sqrt{2(n-1 / 24) / 3}}+1}{2} \\
\leq & \left|G_{n}\right|+\frac{1}{\sqrt{3(n-1 / 24)}}+\sum_{2 \leq l \leq N / 6} \frac{6 \sqrt{l}\left(e^{\frac{\pi}{12} \sqrt{2(n-1 / 24) / 3}}+1\right)}{2 \sqrt{3(n-1 / 24)}} \\
\leq & \left|G_{n}\right|+\sum_{1 \leq l \leq N / 6} \frac{3 \sqrt{l}\left(e^{\frac{\pi}{12}} \sqrt{2(n-1 / 24) / 3}+1\right)}{\sqrt{3(n-1 / 24)}} \\
\leq & \left|G_{n}\right|+\frac{(N / 6)^{3 / 2}\left(3 e^{\frac{\pi}{12} \sqrt{2(n-1 / 24) / 3}}+1\right)}{\sqrt{3(n-1 / 24)}} .
\end{aligned}
$$


Here we have applied (3.20) in the final inequality. Thus,

$$
\begin{aligned}
\left|H_{n}\right| \leq & 1878.5(n-1 / 24)^{1 / 4} \\
& +\frac{\{2 \pi(n-1 / 24)\}^{3 / 4}}{6^{3 / 2}} \frac{3\left(e^{\frac{\pi}{12} \sqrt{2(n-1 / 24) / 3}}+1\right)}{\sqrt{3(n-1 / 24)}} \\
\leq & 0.468(n-1 / 24)^{1 / 4} e^{\frac{\pi}{12} \sqrt{2(n-1 / 24) / 3}}+1879(n-1 / 24)^{1 / 4} .
\end{aligned}
$$

With $H_{n}=E_{2}(n),(2.3)$ follows from (4.31) and (4.33).

4.3. The quotients $\frac{f\left(-q^{6},-q^{10}\right)}{\left(-q^{4} ; q^{4}\right)_{\infty}}, q \frac{f\left(-q^{2},-q^{14}\right)}{\left(-q^{4} ; q^{4}\right)_{\infty}}$. We now estimate $a_{3}(n)$ and $a_{4}(n)$, where

$$
\sum_{n=0}^{\infty} a_{3}(n) q^{n}=\frac{f\left(-q^{6},-q^{10}\right)}{\left(-q^{4} ; q^{4}\right)_{\infty}}, \quad \sum_{n=0}^{\infty} a_{4}(n) q^{n}=\frac{q f\left(-q^{2},-q^{14}\right)}{\left(-q^{4} ; q^{4}\right)_{\infty}}
$$

Note that $a_{3}(2 n+1)=a_{4}(2 n)=0$ for all $n$, since the first quotient contains only even powers of $q$, and the second contains only odd powers of $q$. We may treat both of these simultaneously by considering the sum

$$
S(q):=\frac{f\left(-q^{6},-q^{10}\right)-i q f\left(-q^{2},-q^{12}\right)}{\left(-q^{4} ; q^{4}\right)_{\infty}} .
$$

Replacing $q$ with $i q$ we obtain

$$
S(i q)=\frac{f\left(q^{6}, q^{10}\right)+q f\left(q^{2}, q^{12}\right)}{\left(-q^{4} ; q^{4}\right)_{\infty}} .
$$

By Entry 31 in Chapter 16 of Ramanujan's second notebook [6], we have the following lemma:

LEMMA 4.1. Let $U_{n}=a^{n(n+1) / 2} b^{n(n-1) / 2}$ and $V_{n}=a^{n(n-1) / 2} b^{n(n+1) / 2}$ for each integer $n$. Then

$$
f\left(U_{1}, V_{1}\right)=\sum_{r=0}^{n-1} U_{r} f\left(\frac{U_{n+r}}{U_{r}}, \frac{V_{n-r}}{U_{r}}\right) .
$$

Applying Lemma 4.1 with $(a, b, n)=\left(q, q^{3}, 2\right)$, we find that

$$
f\left(q, q^{3}\right)=f\left(q^{6}, q^{10}\right)+q f\left(q^{14}, q^{2}\right) .
$$

Thus

$$
S:=S(i q)=\frac{f\left(q, q^{3}\right)}{\left(-q^{4} ; q^{4}\right)_{\infty}}=\sum_{n=0}^{\infty} c(n) q^{n}
$$

with

$$
c(n)(q / i)^{n}=a_{3}(n) q^{n}-i a_{4}(n) q^{n} .
$$


Therefore

$$
\begin{aligned}
(-1)^{n} c(2 n) & =a_{3}(2 n), \\
(-1)^{n+1} c(2 n+1) & =a_{4}(2 n+1) .
\end{aligned}
$$

Now we must find an estimate for $c(n)$. To that end, we express $S$ as a quotient of eta-functions and apply the circle method, with $g=S$ in (3.2). Note that, by the Jacobi Triple Product Identity (3.3),

$$
\begin{aligned}
S & =\frac{f\left(q, q^{3}\right)}{\left(-q^{4} ; q^{4}\right)_{\infty}}=\frac{\left(-q ; q^{4}\right)_{\infty}\left(-q^{3} ; q^{4}\right)_{\infty}\left(q^{4} ; q^{4}\right)_{\infty}}{\left(-q^{4} ; q^{4}\right)_{\infty}} \\
& =\frac{\left(q^{2} ; q^{2}\right)_{\infty}^{2}\left(q^{4} ; q^{4}\right)_{\infty}}{(q ; q)_{\infty}\left(q^{8} ; q^{8}\right)_{\infty}}=\frac{F\left(e^{2 \pi i \tau}\right) F\left(e^{2 \pi i(8 \tau)}\right)}{F\left(e^{2 \pi i(2 \tau)}\right)^{2} F\left(e^{2 \pi i(4 \tau)}\right)},
\end{aligned}
$$

with $\tau$ as in (3.10). We consider the cases where $\operatorname{gcd}(k, 8)$ is $1,2,4$, or 8 and apply the transformation formula (3.13) accordingly.

CaSe 1: $k$ is odd. Then

$$
\begin{aligned}
\frac{F\left(e^{2 \pi i \tau}\right) F\left(e^{2 \pi i(8 \tau)}\right)}{F\left(e^{2 \pi i(2 \tau)}\right)^{2} F\left(e^{2 \pi i(4 \tau)}\right)} & \\
= & e^{\pi i\left(s(h, k)+s\left(h_{8}, k\right)-2 s\left(h_{2}, k\right)-s\left(h_{4}, k\right)\right)} e^{\frac{\pi}{12 k}\left(-\frac{1}{8 z}-z\right)} \\
& \times \frac{1}{\sqrt{2 z}} \frac{F\left(e^{2 \pi i \gamma_{(1, k)}(\tau)}\right) F\left(e^{2 \pi i \gamma_{(8, k)}(8 \tau)}\right)}{F\left(e^{2 \pi i \gamma_{(2, k)}(2 \tau)}\right)^{2} F\left(e^{2 \pi i \gamma_{(4, k)}(4 \tau)}\right)} .
\end{aligned}
$$

CASE $2: k=2 k^{\prime}$ with $k^{\prime}$ odd. Then

$$
2^{n} \frac{h+i z}{2 k^{\prime}}=2^{n-1} \frac{h+i z}{k^{\prime}},
$$

whenever $n \geq 1$. Therefore

$$
\begin{aligned}
& \frac{F\left(e^{2 \pi i \tau}\right) F\left(e^{2 \pi i(8 \tau)}\right)}{F\left(e^{2 \pi i(2 \tau)}\right)^{2} F\left(e^{2 \pi i(4 \tau)}\right)} \\
& =e^{\pi i\left(s\left(h, 2 k^{\prime}\right)+s\left(h_{4}, k^{\prime}\right)-2 s\left(h, k^{\prime}\right)-s\left(h_{2}, k^{\prime}\right)\right)} e^{\frac{\pi}{12\left(2 k^{\prime}\right)}\left(-\frac{7}{2 z}-z\right)} \\
& \quad \times \frac{\sqrt{2}}{\sqrt{z}} \frac{F\left(e^{2 \pi i \gamma_{\left(1,2 k^{\prime}\right)}(\tau)}\right) F\left(e^{2 \pi i \gamma_{\left(4, k^{\prime}\right)}(8 \tau)}\right)}{F\left(e^{2 \pi i \gamma_{\left(1, k^{\prime}\right)}(2 \tau)}\right)^{2} F\left(e^{2 \pi i \gamma_{\left(2, k^{\prime}\right)}(4 \tau)}\right)} .
\end{aligned}
$$

CASE $3: k=4 k^{\prime \prime}$ with $k^{\prime \prime}$ odd. Then

$$
\begin{aligned}
& \frac{F\left(e^{2 \pi i \tau}\right) F\left(e^{2 \pi i(8 \tau)}\right)}{F\left(e^{2 \pi i(2 \tau)}\right)^{2} F\left(e^{2 \pi i(4 \tau)}\right)} \\
& =e^{\pi i\left(s\left(h, 4 k^{\prime \prime}\right)+s\left(h_{2}, k^{\prime \prime}\right)-2 s\left(h, 2 k^{\prime \prime}\right)-s\left(h, k^{\prime \prime}\right)\right)} e^{\frac{\pi}{12\left(4 k^{\prime \prime}\right)}\left(-\frac{7}{z}-z\right)} \\
& \quad \times \frac{\sqrt{2}}{\sqrt{z}} \frac{F\left(e^{2 \pi i \gamma_{\left(1,4 k^{\prime \prime}\right)}(\tau)}\right) F\left(e^{2 \pi i \gamma_{\left(2, k^{\prime \prime}\right)}(8 \tau)}\right)}{F\left(e^{2 \pi i \gamma_{\left(1,2 k^{\prime \prime}\right)}(2 \tau)}\right)^{2} F\left(e^{2 \pi i \gamma_{\left(1, k^{\prime \prime}\right)}(4 \tau)}\right)}
\end{aligned}
$$


CASE $4: k=8 l$. Then

$$
\begin{aligned}
& \frac{F\left(e^{2 \pi i \tau}\right) F\left(e^{2 \pi i(8 \tau)}\right)}{F\left(e^{2 \pi i(2 \tau)}\right)^{2} F\left(e^{2 \pi i(4 \tau)}\right)} \\
& \quad=\omega_{h, l} e^{\frac{\pi}{12(8 l)}\left(\frac{1}{z}-z\right)} \frac{1}{\sqrt{z}} \frac{F\left(e^{2 \pi i \gamma_{(1,8 l)}(\tau)}\right) F\left(e^{2 \pi i \gamma_{(1, l)}(8 \tau)}\right)}{F\left(e^{2 \pi i \gamma_{(1,4 l)}(2 \tau)}\right)^{2} F\left(e^{2 \pi i \gamma_{(1,2 l)}(4 \tau)}\right)},
\end{aligned}
$$

with

$$
\omega_{h, l}^{\prime \prime}=e^{\pi i(s(h, 8 l)+s(h, l)-2 s(h, 4 l)-s(h, 2 l))} .
$$

We subdivide the integral for $c(n)$ into four pieces corresponding to each of these four cases to obtain

$$
\begin{aligned}
c(n)= & \left(\sum_{\substack{1 \leq k \leq N \\
k \text { odd }}}+\sum_{\substack{1 \leq k \leq N \\
k \equiv 2(\bmod 4)}}+\sum_{\substack{1 \leq k \leq N \\
k \equiv 4(\bmod 8)}}+\sum_{\substack{1 \leq k \leq N \\
k \equiv 0(\bmod 8)}}\right) \\
& \times \sum_{\substack{0 \leq h \leq k \\
(h, k)=1}} e^{-2 \pi i n h / k} \int_{\xi_{h, k}} \frac{F\left(e^{2 \pi i \tau}\right) F\left(e^{2 \pi i(8 \tau)}\right)}{F\left(e^{2 \pi i(2 \tau)}\right)^{2} F\left(e^{2 \pi i(4 \tau)}\right)} e^{2 \pi n \varrho} e^{-2 \pi i n \varphi} d \varphi \\
= & : S_{1}\left(A_{1}\right)+S_{2}\left(A_{2}\right)+S_{3}\left(A_{3}\right)+S_{4}\left(A_{4}\right),
\end{aligned}
$$

where

$$
A_{j}=\frac{F\left(e^{2 \pi i \tau}\right) F\left(e^{2 \pi i(8 \tau)}\right)}{F\left(e^{2 \pi i(2 \tau)}\right)^{2} F\left(e^{2 \pi i(4 \tau)}\right)} e^{2 \pi n \varrho} e^{-2 \pi i n \varphi}
$$

for $j=1,2,3,4$. Applying (4.39) to $A_{1},(4.40)$ to $A_{2}$, and (4.41) to $A_{3}$, and then using $(3.11),(3.12),(3.16),(3.18)$, and $(3.19)$, we find that

$$
\begin{aligned}
\left|A_{1}\right| \leq & e^{-\frac{2 \pi}{k} \operatorname{Re}\left(\frac{1}{192 z}\right)} e^{-\frac{\pi}{12 k} \operatorname{Re}(z)} \\
& \times \frac{1}{\sqrt{2}}|z|^{-1 / 2}\left|\frac{F\left(e^{-\frac{2 \pi}{k} \operatorname{Re}\left(\frac{1}{z}\right)}\right) F\left(e^{-\frac{2 \pi}{k} \operatorname{Re}\left(\frac{1}{8 z}\right)}\right)}{F\left(e^{-\frac{2 \pi}{k} \operatorname{Re}\left(\frac{1}{2 z}\right)}\right)^{2} F\left(e^{-\frac{2 \pi}{k} \operatorname{Re}\left(\frac{1}{4 z}\right)}\right)}\right| e^{2 \pi n \varrho} \\
\leq & \frac{e^{8.638631}}{\sqrt{2}} \frac{e^{2 \pi(n-1 / 24) \varrho} N}{k^{1 / 2}} \\
\left|A_{2}\right| \leq & e^{-\frac{2 \pi}{2 k^{\prime}} \operatorname{Re}\left(\frac{7}{48 z}\right)} e^{-\frac{\pi}{12\left(2 k^{\prime}\right)} \operatorname{Re}(z)} \\
& \times \sqrt{2}|z|^{-1 / 2}\left|\frac{F\left(e^{-\frac{2 \pi}{k} \operatorname{Re}\left(\frac{1}{z}\right)}\right) F\left(e^{-\frac{4 \pi}{k} \operatorname{Re}\left(\frac{1}{4 z}\right)}\right)}{F\left(e^{-\frac{4 \pi}{k} \operatorname{Re}\left(\frac{1}{z}\right)}\right)^{2} F\left(e^{-\frac{4 \pi}{k} \operatorname{Re}\left(\frac{1}{2 z}\right)}\right)}\right| e^{2 \pi n \varrho} \\
\leq & \frac{e^{-0.02868} \sqrt{2} N}{k^{1 / 2}} e^{2 \pi(n-1 / 24) \varrho}
\end{aligned}
$$




$$
\begin{aligned}
\left|A_{3}\right| \leq & e^{-\frac{2 \pi}{k} \operatorname{Re}\left(\frac{7}{24 z}\right)} e^{-\frac{\pi}{12 k} \operatorname{Re}(z)} \\
& \times \sqrt{2}|z|^{-1 / 2}\left|\frac{F\left(e^{-\frac{2 \pi}{k} \operatorname{Re}\left(\frac{1}{z}\right)}\right) F\left(e^{-\frac{8 \pi}{k} \operatorname{Re}\left(\frac{1}{2 z}\right)}\right)}{F\left(e^{-\frac{4 \pi}{k} \operatorname{Re}\left(\frac{1}{z}\right)}\right)^{2} F\left(e^{-\frac{8 \pi}{k} \operatorname{Re}\left(\frac{1}{z}\right)}\right)}\right| e^{2 \pi n \varrho} \\
\leq & \frac{e^{-0.863} \sqrt{2} N}{k^{1 / 2}} e^{2 \pi(n-1 / 24) \varrho} .
\end{aligned}
$$

Combining (4.45)-(4.47) with (3.15), we deduce that

$$
\begin{aligned}
& \left|S_{1}\left(A_{1}\right)\right|+\left|S_{2}\left(A_{2}\right)\right|+\left|S_{3}\left(A_{3}\right)\right| \\
& \leq \sum_{\substack{1 \leq k \leq N \\
k \text { odd }}} \sum_{\substack{0 \leq h \leq k \\
(h, k)=1}} \frac{2}{k N} \frac{e^{8.638631}}{\sqrt{2}} \frac{e^{2 \pi(n-1 / 24) \varrho} N}{k^{1 / 2}} \\
& \quad+\sum_{\substack{1 \leq k \leq N \\
k \equiv 2(\bmod 4)}} \sum_{\substack{0 \leq h \leq k \\
(h, k)=1}} \frac{2}{k N} \frac{e^{-0.02868} \sqrt{2} N}{k^{1 / 2}} e^{2 \pi(n-1 / 24) \varrho} \\
& \quad+\sum_{\substack{1 \leq k \leq N \\
k \equiv 4(\bmod 8)}} \sum_{\substack{0 \leq h \leq k \\
(h, k)=1}} \frac{2}{k N} \frac{e^{-0.863} \sqrt{2} N}{k^{1 / 2}} e^{2 \pi(n-1 / 24) \varrho} \\
& \leq \sum_{1 \leq k \leq N}\left(\sqrt{2} e^{8.638631}+2 \sqrt{2} e^{-0.02868}+2 \sqrt{2} e^{-0.863}\right) e^{2 \pi(n-1 / 24) \varrho} k^{-1 / 2} .
\end{aligned}
$$

Using (3.20), we find that

$$
\begin{aligned}
\left|S_{1}\left(A_{1}\right)\right| & +\left|S_{2}\left(A_{2}\right)\right|+\left|S_{3}\left(A_{3}\right)\right| \\
\leq & 2 \sqrt{2}\left(e^{8.638631}+2 e^{-0.02868}+2 e^{-0.863}\right) e^{2 \pi(n-1 / 24) \varrho} \sqrt{N} .
\end{aligned}
$$

To estimate $S_{4}$, set $k=8 l$, so that we have $\tau=(h+i z) / 8 l$ and $z=$ $(8 l)(\varrho-i \varphi)$. Therefore, when we apply the transformation formula (4.42), we obtain

$$
\begin{aligned}
S_{4}\left(A_{4}\right)= & \sum_{1 \leq 8 l \leq N} \sum_{\substack{0 \leq h \leq 8 l \\
(h, 8 l)=1}} e^{-2 \pi i n h / 8 l} \int_{\xi_{h, 8 l}} \omega_{h, l}^{\prime \prime} e^{\frac{\pi}{12(8 l)}\left(\frac{1}{z}-z\right)} \\
& \times \frac{1}{\sqrt{z}} \frac{F\left(e^{2 \pi i \gamma_{(1,8 l)}(\tau)}\right) F\left(e^{2 \pi i \gamma_{(1, l)}(8 \tau)}\right)}{F\left(e^{2 \pi i \gamma_{(1,4 l)}(2 \tau)}\right)^{2} F\left(e^{2 \pi i \gamma_{(1,2 l)}(4 \tau)}\right)} e^{2 \pi n \varrho} e^{-2 \pi i n \varphi} d \varphi .
\end{aligned}
$$

Set

$$
\tau^{\prime}:=\frac{h^{\prime}+i z^{-1}}{8 l}
$$

so that for $n=1,2,4,8$,

$$
\gamma_{(1,8 l / n)}(n \tau)=n \tau^{\prime}
$$


Thus,

$$
\begin{aligned}
S_{4}\left(A_{4}\right)= & \sum_{1 \leq 8 l \leq N} \sum_{\substack{0 \leq h \leq 8 l \\
(h, 8 l)=1}} e^{-2 \pi i n h / 8 l} \int_{\xi_{h, 8 l}} \omega_{h, l}^{\prime \prime} e^{\frac{\pi}{12(8 l)}\left(\frac{1}{z}-z\right)} \\
& \times \frac{1}{\sqrt{z}} \frac{F\left(e^{2 \pi i \tau^{\prime}}\right) F\left(e^{2 \pi i\left(8 \tau^{\prime}\right)}\right)}{F\left(e^{2 \pi i\left(2 \tau^{\prime}\right)}\right)^{2} F\left(e^{2 \pi i\left(4 \tau^{\prime}\right)}\right)} e^{2 \pi n \varrho} e^{-2 \pi i n \varphi} d \varphi \\
= & \sum_{1 \leq 8 l \leq N} \sum_{\substack{0 \leq h \leq 8 l \\
(h, 8 l)=1}} e^{-2 \pi i n h / 8 l} \\
& \times \int_{\xi_{h, 8 l}} \omega_{h, l}^{\prime \prime} e^{\frac{\pi}{12(8 l)}\left(\frac{1}{z}-z\right)} \frac{1}{\sqrt{z}} e^{2 \pi n \varrho} e^{-2 \pi i n \varphi} d \varphi \\
& +\sum_{1 \leq 8 l \leq N} \sum_{\substack{0 \leq h \leq 8 l \\
(h, 8 l)=1}} e^{-2 \pi i n h / 8 l} \int_{h, l}^{\prime \prime} e^{\frac{\pi}{12(8 l)}\left(\frac{1}{z}-z\right)} \\
& \times \frac{1}{\sqrt{z}}\left(\frac{F\left(e^{2 \pi i \tau^{\prime}}\right) F\left(e^{2 \pi i\left(8 \tau^{\prime}\right)}\right)}{F\left(e^{2 \pi i\left(2 \tau^{\prime}\right)}\right)^{2} F\left(e^{2 \pi i\left(4 \tau^{\prime}\right)}\right)}-1\right) e^{2 \pi n \varrho} e^{-2 \pi i n \varphi} d \varphi \\
=: & T_{1}+T_{2} . \quad
\end{aligned}
$$

Let $P_{n}$ be the set of partitions of $n$ where each part congruent to $4(\bmod 8)$ appears at most twice, and where the even parts not congruent to $4(\bmod 8)$ are distinct. For example, the partition $12+12+8+3+3+3+2 \in P_{43}$ but $12+8+6+6+3+3+3+2 \notin P_{43}$. Since

$$
S=\frac{\left(q^{2} ; q^{2}\right)_{\infty}^{2}\left(q^{4} ; q^{4}\right)_{\infty}}{(q ; q)_{\infty}\left(q^{8} ; q^{8}\right)_{\infty}}=\frac{\left(q^{2} ; q^{2}\right)_{\infty}\left(q^{4} ; q^{8}\right)_{\infty}}{\left(q ; q^{2}\right)_{\infty}}
$$

we see that $c(n)$ represents the number of partitions in $P_{n}$ with an even number of even parts minus the number of partitions in $P_{n}$ with an odd number of even parts. Thus, $\left|c_{n}\right| \leq p(n)$, the ordinary partition function. Therefore, we have

$$
\begin{aligned}
\left|T_{2}\right| \leq & \sum_{1 \leq 8 l \leq N} \sum_{\substack{0 \leq h \leq 8 l \\
(h, 8 l)=1}} \int_{\xi_{h, 8 l}} e^{\frac{2 \pi}{24(8 l)} \operatorname{Re}\left(\frac{1}{z}\right)} e^{-\frac{\pi}{12(8 l)} \operatorname{Re}(z)}|z|^{-1 / 2} \\
& \times\left|F\left(\left|e^{2 \pi i \tau^{\prime}}\right|\right)-1\right| e^{2 \pi n \varrho} d \varphi
\end{aligned}
$$

Let $x=\left|e^{2 \pi i \tau^{\prime}}\right|=e^{-\frac{2 \pi}{8 l} \operatorname{Re}\left(\frac{1}{z}\right)}$. Then (3.16) implies that $x \leq e^{-\pi}$. Since $x^{-1 / 24}|F(x)-1|$ is an increasing function of $x$, it is maximized at $x=e^{-\pi}$. Apply (3.19) to obtain

$$
x^{-1 / 24}|F(x)-1| \leq 0.0551 .
$$


Using this in conjunction with (3.18), we find that

$$
\begin{aligned}
\left|T_{2}\right| & \leq \sum_{1 \leq 8 l \leq N} \sum_{\substack{0 \leq h \leq 8 l \\
(h, 8 l)=1}} \int_{\xi_{h, 8 l}} 0.0551 \frac{N}{(8 l)^{1 / 2}} e^{2 \pi(n-1 / 24) \varrho} d \varphi \\
& \leq \sum_{1 \leq 8 l \leq N} \sum_{\substack{0 \leq h \leq 8 l \\
(h, 8 l)=1}} 0.0551 \frac{2}{(8 l)^{3 / 2}} e^{2 \pi(n-1 / 24) \varrho} \\
& \leq \sum_{1 \leq 8 l \leq N} 0.1102 \frac{1}{(8 l)^{1 / 2}} e^{2 \pi(n-1 / 24) \varrho} \\
& \leq 0.2204 e^{2 \pi(n-1 / 24) \varrho \sqrt{N}} .
\end{aligned}
$$

Applying (3.21) and (3.22) to $T_{1}$ with $k=8 l$, we obtain

$$
\begin{aligned}
T_{1}= & D_{n}+\sum_{\substack { 1 \leq 8 l \leq N \\
\begin{subarray}{c}{0 \leq h \leq 8 l \\
(h, 8 l)=1{ 1 \leq 8 l \leq N \\
\begin{subarray} { c } { 0 \leq h \leq 8 l \\
( h , 8 l ) = 1 } }\end{subarray}} e^{-2 \pi i n h / 8 l} \omega_{h, l}^{\prime \prime} \\
& \times \sqrt{\frac{2}{8 l(n-1 / 24)}} \cosh \left(\frac{\pi}{8 l} \sqrt{\frac{2}{3}(n-1 / 24)}\right),
\end{aligned}
$$

where

$$
\begin{aligned}
\left|D_{n}\right| & \leq \sum_{1 \leq 8 l \leq N} \sum_{\substack{0 \leq h \leq 8 l \\
(h, 8 l)=1}} 1.2828 \frac{e^{2 \pi(n-1 / 24) \varrho} N^{1 / 2}}{n-1 / 24} \\
& \leq \sum_{1 \leq 8 l \leq N} 1.2828 \frac{e^{2 \pi(n-1 / 24) \varrho(8 l) N^{1 / 2}}}{n-1 / 24} \\
& \leq 1.2828 \frac{e^{2 \pi(n-1 / 24) \varrho N^{5 / 2}}}{n-1 / 24} .
\end{aligned}
$$

Combining (4.48) and (4.51)-(4.53), we find that

$$
c(n)=\sum_{1 \leq 8 l \leq N} \sum_{\substack{0 \leq h \leq 8 l \\(h, 8 l)=1}} \frac{e^{-2 \pi i n h / 8 l} \omega_{h, l}^{\prime \prime}}{\sqrt{4 l(n-1 / 24)}} \cosh \left(\frac{\pi}{8 l} \sqrt{\frac{2}{3}(n-1 / 24)}\right)+G_{n},
$$

with

$$
\begin{aligned}
\left|G_{n}\right| \leq & \left|S_{1}\left(A_{1}\right)\right|+\left|S_{2}\left(A_{2}\right)\right|+\left|S_{3}\left(A_{3}\right)\right|+\left|T_{2}\right|+\left|D_{n}\right| \\
\leq & 2 \sqrt{2}\left(e^{8.638631}+2 e^{-0.02868}+2 e^{-0.863}\right) e^{2 \pi(n-1 / 24) \varrho \sqrt{N}} \\
& +0.2204 e^{2 \pi(n-1 / 24) \varrho \sqrt{N}}+1.2828 \frac{e^{2 \pi(n-1 / 24) \varrho} N^{5 / 2}}{n-1 / 24} .
\end{aligned}
$$


Choose $N=\sqrt{2 \pi(n-1 / 24)}$, so that $\varrho=N^{-2}=\frac{1}{2 \pi(n-1 / 24)}$. Then

$$
\begin{aligned}
\left|G_{n}\right| \leq & e\left(2 \sqrt{2}\left(e^{8.638631}+2 e^{-0.02868}+2 e^{-0.863}\right)+0.2204+(2 \pi) 1.2828\right) \\
& \times(2 \pi)^{1 / 4}(n-1 / 24)^{1 / 4} \\
\leq & 68792(n-1 / 24)^{1 / 4}
\end{aligned}
$$

We now approximate the sum with the $l=1$ term, and find an upper bound for the remaining terms. Therefore, for $n \geq 1$,

$$
c(n)=\sum_{\substack{0 \leq h \leq 8 \\(h, 8)=1}} e^{-2 \pi i n h / 8} \omega_{h, 1}^{\prime \prime} \frac{1}{\sqrt{4(n-1 / 24)}} \frac{e^{\frac{\pi}{8} \sqrt{2(n-1 / 24) / 3}}}{2}+H_{n}
$$

with

$$
\begin{aligned}
H_{n}=G_{n} & +\sum_{\substack{0 \leq h \leq 8 \\
(h, 8)=1}} \frac{e^{-2 \pi i n h / 8} \omega_{h, 1}^{\prime \prime}}{\sqrt{4(n-1 / 24)}} \frac{e^{-\frac{\pi}{8} \sqrt{2(n-1 / 24) / 3}}}{2} \\
& +\sum_{2 \leq l \leq N / 8} \sum_{\substack{0 \leq h \leq 8 l \\
(h, 8 l)=1}} \frac{e^{-2 \pi i n h / 8 l} \omega_{h, l}^{\prime \prime}}{\sqrt{4 l(n-1 / 24)}} \frac{e^{\frac{\pi}{8 l} \sqrt{2(n-1 / 24) / 3}}+e^{-\frac{\pi}{8 l} \sqrt{2(n-1 / 24) / 3}}}{2}
\end{aligned}
$$

We compute $\sum_{0 \leq h \leq 8,(h, 8)=1} e^{-2 \pi i n h / 8} \omega_{h, 1}^{\prime \prime}$ using (3.7) and (3.8). Since

$$
\omega_{h, l}^{\prime \prime}=e^{\pi i(s(h, 8 l)+s(h, l)-2 s(h, 4 l)-s(h, 2 l))},
$$

we find that

$$
\omega_{1,1}^{\prime \prime}=e^{3 \pi i / 16}, \quad \omega_{3,1}^{\prime \prime}=e^{5 \pi i / 16}, \quad \omega_{5,1}^{\prime \prime}=e^{-5 \pi i / 16}, \quad \omega_{7,1}^{\prime \prime}=e^{-3 \pi i / 16} .
$$

Therefore,

$$
\sum_{\substack{0 \leq h \leq 8 \\(h, 8)=1}} e^{-2 \pi i n h / 8} \omega_{h, 1}^{\prime \prime}= \begin{cases}2(\cos (3 \pi / 16)+\cos (5 \pi / 16)) & \text { if } n \equiv 0(\bmod 8), \\ 2(\cos (\pi / 16)+\cos (7 \pi / 16)) & \text { if } n \equiv 1(\bmod 8), \\ -2(\cos (3 \pi / 16)-\cos (5 \pi / 16)) & \text { if } n \equiv 2(\bmod 8), \\ 2(\cos (\pi / 16)-\cos (7 \pi / 16)) & \text { if } n \equiv 3(\bmod 8), \\ -2(\cos (3 \pi / 16)+\cos (5 \pi / 16)) & \text { if } n \equiv 4(\bmod 8), \\ -2(\cos (\pi / 16)+\cos (7 \pi / 16)) & \text { if } n \equiv 5(\bmod 8), \\ 2(\cos (3 \pi / 16)-\cos (5 \pi / 16)) & \text { if } n \equiv 6(\bmod 8), \\ -2(\cos (\pi / 16)-\cos (7 \pi / 16)) & \text { if } n \equiv 7(\bmod 8)\end{cases}
$$


Bounding the terms involving the negative exponents with $l=1$, and the positive exponents at $l=2$, we see that

$$
\begin{aligned}
\left|H_{n}\right| \leq & \left|G_{n}\right|+\frac{1}{\sqrt{n-1 / 24}} \\
& +\sum_{2 \leq l \leq N / 8} \sum_{\substack{0 \leq h \leq 8 l \\
(h, 8 l)=1}} \frac{1}{\sqrt{l(n-1 / 24)}} \frac{e^{\frac{\pi}{16}} \sqrt{2(n-1 / 24) / 3}+1}{4} \\
\leq & \left|G_{n}\right|+\frac{1}{\sqrt{n-1 / 24}} \\
& +\sum_{2 \leq l \leq N / 8} \frac{\sqrt{l}}{\sqrt{(n-1 / 24)}}\left(e^{\frac{\pi}{16} \sqrt{2(n-1 / 24) / 3}}+1\right) \\
\leq & \left|G_{n}\right|+\sum_{1 \leq l \leq N / 8} \frac{\sqrt{l}}{\sqrt{n-1 / 24}}\left(e^{\frac{\pi}{16} \sqrt{2(n-1 / 24) / 3}}+1\right) \\
\leq & \left|G_{n}\right|+\frac{(N / 8)^{3 / 2}}{\sqrt{n-1 / 24}}\left(e^{\frac{\pi}{16} \sqrt{2(n-1 / 24) / 3}}+1\right) \\
\leq & \left|G_{n}\right|+\frac{(2 \pi)^{3 / 4}(n-1 / 24)^{1 / 4}}{8^{3 / 2}}\left(e^{\frac{\pi}{16}} \sqrt{2(n-1 / 24) / 3}+1\right) \\
\leq & 68793(n-1 / 24)^{1 / 4}+0.1754(n-1 / 24)^{1 / 4} e^{\frac{\pi}{16}} \sqrt{2(n-1 / 24) / 3}
\end{aligned}
$$

With $H_{n}=E_{3}(n)$, equalities (2.4) and (2.5) follow from (4.36), (4.37), (4.54), and (4.56). The proof of Theorem 2.1 is complete.

Acknowledgements. The author would like to thank Professor Bruce Berndt for suggesting this problem and for his many comments as well as his guidance on this paper.

\section{References}

[1] G. E. Andrews, The Theory of Partitions, Cambridge Univ. Press, Cambridge, 1984.

[2] G. E. Andrews and F. G. Garvan, Dyson's crank of a partition, Bull. Amer. Math. Soc. (N.S.) 18 (1988), 167-171.

[3] G. E. Andrews and R. Lewis, The ranks and cranks of partitions moduli 2, 3, and 4, J. Number Theory 85 (2000), 74-84.

[4] T. M. Apostol, Modular Functions and Dirichlet Series in Number Theory, 2nd ed., Grad. Texts in Math. 41, Springer, New York, 1990.

[5] A. O. L. Atkin and H. P. F. Swinnerton-Dyer, Some properties of partitions, Proc. London Math. Soc. (3) 4 (1954), 84-106.

[6] B. C. Berndt, Ramanujan's Notebooks, Part III, Springer, New York, 1991. 
[7] B. C. Berndt, H. H. Chan, S. H. Chan and W.-C. Liaw, Ramanujan and cranks, in: Theory and Applications of Special Functions. A volume dedicated to Mizan Rahman, M. E. H. Ismail and E. Koelink (eds.), Dev. Math. 13, Springer, New York, 2005, 77-98.

[8] F. J. Dyson, Some guesses in the theory of partitions, Eureka (Cambridge) 8 (1944), $10-15$.

[9] G. H. Hardy and E. M. Wright, An Introduction to the Theory of Numbers, 3rd ed., Oxford Univ. Press, London, 1954.

[10] D. M. Kane, Resolution of a conjecture of Andrews and Lewis involving cranks of partitions, Proc. Amer. Math. Soc. 132 (2004), 2247-2256.

[11] S. Ramanujan, The Lost Notebook and Other Unpublished Papers, Narosa, New Delhi, 1988.

[12] E. T. Whittaker and G. N. Watson, A Course of Modern Analysis, 4th ed., Cambridge Univ. Press, Cambridge, 1952.

Department of Mathematics

University of Illinois at Urbana-Champaign

1409 West Green Street

Urbana, IL 61801, U.S.A.

E-mail: ochan@math.uiuc.edu

Received on 16.7.2004

and in revised form on 9.7.2005 